\title{
Benzoic acid-promoted C2-H Borylation of Indoles with Pinacolborane
}

Youliang Zou, Binfeng Zhang, Li Wang, Hua Zhang*

Key Laboratory of Catalysis and Energy Materials Chemistry of Ministry of Education and Hubei Key Laboratory of Catalysis and Materials Science, School of Chemistry and Materials Science, South-Central University for Nationalities, Wuhan 430074 (China) and College of Chemistry, Nanchang University, Nanchang 330031 (China) huazhang@sscuec.edu.cn

\section{Supplementary Information}

\section{Table of Contents}

1. General information

2. Reaction optimization

3. Benzoic acid-promoted $\mathrm{C} 2-\mathrm{H}$ borylation of indoles with pinacolborane $\mathrm{S} 4$

4. Transformation of 2-boryl indoles

5. Control experiments

6. The ${ }^{11} \mathrm{~B}$ NMR analysis of the borylation reaction system

7. Monitoring of the borylation of 1a with pinacolborane

8. Analytical data of products

9. Reference

10. NMR spectra of products 


\section{General information}

Unless otherwise noted, all reactants or reagents were obtained from commercial suppliers and used as received. Benzoic acid, pinacolborane, 3,4,5-trichloropyridine were obtained from Energy Chemical. Acetic acid was obtained from Sinopharm. $\mathrm{BH}_{3} \cdot \mathrm{DMS}$ (2.0 M in DMS), $\mathrm{BH}_{3} \cdot \mathrm{THF}$ (1.0 M in THF), $\mathrm{BH}_{3} \bullet$ py and $\mathrm{BH}_{3} \bullet \mathrm{TMA}$ were obtained from Energy Chemical (Shanghai, China). $\mathrm{LiAlH}_{4}(1.0$ $\mathrm{M}$ in THF) was obtained from Adamas. ${ }^{c}$ Hexane was obtained from Sinopharm and purified by distillation with sodium. $\mathrm{CDCl}_{3}$ was obtained from $\mathrm{JK}$ Chemical. Otherwise noted, all reactions were performed with dry solvents under an atmosphere of nitrogen gas (99.999\%) in dried glassware using standard Schlenk techniques. All $N$-alkyl indoles ${ }^{[1-4]}$ were prepared according to the procedures reported in the literature. Starting materials $\mathbf{1 i},{ }^{[5]} \mathbf{1} \mathbf{j},{ }^{[6]}$ and $\mathbf{1 0}{ }^{[7]}$ are known compounds and were prepared according to the procedures reported in the literature. $\mathrm{BnOBpin}{ }^{[8]}$ was prepared according to the procedures reported in the literature. All reactions were performed in a $25-\mathrm{mL}$ Schlenk tube (with a Teflon high-pressure valve and side arm) and heated in a heating module (heater + magnetic stirrer). All work-up and purification procedures were carried out with reagent-grade solvents in air.

Analytical thin-layer chromatography (TLC) was performed using Huang Hai HSGF254 (0.2 $\mathrm{mm})$ precoated plates. The developed chromatogram was analyzed by UV lamp (254 $\mathrm{nm})$. Flash column chromatography was performed with silica gel (200-300 mesh). GCMS analysis was conducted on a Shimadzu GCMS-QP2010 instrument equipped with a Restec-5HT column (30 m $\times$ $0.25 \mathrm{~mm}$, Hewlett-Packard). Nuclear magnetic resonance (NMR) spectra was recorded on Bruker Advance III (400 MHz) spectrometers with tetramethyl silane as an internal standard. Chemical shifts for ${ }^{1} \mathrm{H}$ NMR are expressed in parts per million ( $\left.\mathrm{ppm}\right)$ relative to tetramethyl silane $(\delta 0.00 \mathrm{ppm})$ or residual peak of $\mathrm{CDCl}_{3}(\delta 7.26 \mathrm{ppm})$. Chemical shifts for ${ }^{13} \mathrm{C}$ NMR are expressed in ppm relative to $\mathrm{CDCl}_{3}(\delta 77.16 \mathrm{ppm})$. Data are reported as follows: chemical shift, multiplicity (s = singlet, $\mathrm{d}=$ doublet, $\mathrm{dd}=$ doublet of doublets, $\mathrm{t}=$ triplet, $\mathrm{m}=$ multiplet $)$, coupling constant $(\mathrm{Hz})$, and integration. High resolution mass spectra (HRMS) were obtained from Agilent 6545 Q-TOF LCMS with electrospray ionization (ESI). 


\section{Reaction optimization}

An oven-dried $25-\mathrm{mL}$ Schlenk tube equipped with a magnetic stirring bar was charged with 1a (0.4 mmol, $52.5 \mathrm{mg})$, HBpin $(2.4 \mathrm{mmol}, 307.2 \mathrm{mg})$, promoter $(0.04 \mathrm{mmol})$ and solvent $(1.0 \mathrm{~mL})$ under nitrogen atmosphere. The sealed tube was heated in a heating module with stirring. After cooling the reaction mixture to room temperature, the solvent was evaporated under reduced pressure. The yields of 2a, 3a and $4 \mathbf{a}$ were determined by ${ }^{1} \mathrm{H}$ NMR using 3,4,5-trichloropyridine as internal standard.

Note: $\mathbf{4 a}$ was detected by GC, GCMS, HRMS and crude NMR. 4a is not particularly stable and easily undergoes C3-protodeborylation under acidic conditions or on silica gel to afford 2a quantitatively. Therefore, it is difficult to obtain pure 4a. The HRMS information provided convenience for further determining the structure of 4a: HRMS (ESI-TOF) m/z: $[\mathrm{M}+\mathrm{H}]^{+}$Calcd for $\mathrm{C}_{21} \mathrm{H}_{32} \mathrm{~B}_{2} \mathrm{NO}_{4}$ 384.2512; Found 384.2521. The corresponding C2,C3-diborylated indoles were also detected by GCMS in the reaction of $\mathbf{1 d}, \mathbf{1 e}, \mathbf{1 f}, \mathbf{1 g}$ and other substrates. 


\section{Benzoic acid-catalyzed C2-H borylation of indoles with pinacolborane}

General Procedure: An oven-dried 25-mL Schlenk-type sealed tube equipped with a magnetic stirring bar was charged with benzoic acid $(0.04 \mathrm{mmol}, 4.9 \mathrm{mg})$, and then flushed with nitrogen gas. 1 (0.4 mmol), HBpin (2.4 mmol, $307.2 \mathrm{mg})$, and chexane $(1.0 \mathrm{~mL})$ were added to the tube under nitrogen atmosphere. The sealed tube was heated at $180^{\circ} \mathrm{C}$ for $16 \mathrm{~h}$ in a heating module with stirring. After cooling the reaction mixture to room temperature, the mixture was acidified with $6.0 \mathrm{M} \mathrm{HCl}$ aqueous solution $(1.0 \mathrm{~mL})$ and stirred for $0.5 \mathrm{~h}$. Then the mixture was extracted with ethyl acetate and dried over $\mathrm{Na}_{2} \mathrm{SO}_{4}$. The solvent was evaporated under reduced pressure and the residue was directly purified by flash column chromatography over silica gel eluting with petroleum ether/ethyl acetate to afford the product.

Note: The reaction of $1 \mathbf{p}$ was conducted with $\mathrm{CH}_{3} \mathrm{CO}_{2} \mathrm{H}(0.04 \mathrm{mmol}, 2.4 \mathrm{mg})$. The reaction mixture was not acidified with $\mathrm{HCl}$ aqueous solution for the reaction of $\mathbf{1 i}, \mathbf{1 q}$ and $\mathbf{1 r}$.

Hazardous statement: These reactions were performed at a temperature that is well above the boiling point of ${ }^{c}$ hexane (approximately $80{ }^{\circ} \mathrm{C}$ ), leading to substantial pressure buildup in the sealed reaction vessel. As such, proper precautions should be taken when performing these experiments. All reactions were performed in a $25-\mathrm{mL}$ Schlenk tube (with a Teflon high-pressure valve and side arm) and heated in a heating module (heater + magnetic stirrer).

General Procedure (1mmol scale): An oven-dried 25-mL Schlenk tube equipped with a magnetic stirring bar was charged with benzoic acid $(0.1 \mathrm{mmol}, 12.2 \mathrm{mg})$, and then flushed with nitrogen gas. 1a $(1.0 \mathrm{mmol}, 131.2 \mathrm{mg})$, HBpin $(6.0 \mathrm{mmol}, 767.9 \mathrm{mg})$, and ${ }^{c}$ hexane $(2.5 \mathrm{~mL})$ were added to the tube under nitrogen atmosphere. The sealed tube was heated at $180{ }^{\circ} \mathrm{C}$ for $16 \mathrm{~h}$ in a heating module with stirring. After cooling the reaction mixture to room temperature, the mixture was added $6.0 \mathrm{M} \mathrm{HCl}$ aqueous solution $(1.0 \mathrm{~mL})$ and stirred for $0.5 \mathrm{~h}$. Then the mixture was extracted with ethyl acetate and dried over $\mathrm{Na}_{2} \mathrm{SO}_{4}$. The solvent was evaporated under reduced pressure and the residue was directly purified by flash column chromatography over silica gel (eluent: petroleum ether/ethyl acetate = 200:1) to afford $\mathbf{2 a}(169.7 \mathrm{mg}, 66 \%$ yield) as white solid. 


\section{Transformation of 2-boryl indoles}

Compound 5 was prepared according to the procedures reported in the literature. ${ }^{\left[{ }^{9]}\right.} \mathbf{2 a}(0.2 \mathrm{mmol}$, $51.4 \mathrm{mg})$ was dissolved in THF/Et $2 \mathrm{O}(1: 1 \mathrm{v} / \mathrm{v}, 4.5 \mathrm{~mL})$ and cooled to $0{ }^{\circ} \mathrm{C}$. A $2.0 \mathrm{M} \mathrm{NaOH} / 30 \% \mathrm{H}_{2} \mathrm{O}_{2}$ $(2: 1 \mathrm{v} / \mathrm{v}, 1.5 \mathrm{~mL})$ mixture was added dropwise and the reaction was allowed to warm to RT and then stirred for $2 \mathrm{~h}$. The mixture was diluted with $\mathrm{H}_{2} \mathrm{O}(10 \mathrm{~mL})$, the layers were separated, and the aqueous layer was extracted with EtOAc. The combined organic layers were washed with saturated brines, dried over $\mathrm{Na}_{2} \mathrm{SO}_{4}$, filtered, and concentrated by rotary evaporation. The crude mixture was purified by column chromatography on silica gel to afford the corresponding product $\mathbf{5}(23.8 \mathrm{mg}, 81 \%)$ as a white solid.

Compound 6 was prepared according to the modified procedures reported in the literature. ${ }^{[10]}$ An oven-dried $25-\mathrm{mL}$ Schlenk tube equipped with a magnetic stirring bar was charged with $\mathrm{Pd}\left(\mathrm{PPh}_{3}\right)_{4}(5.8$ $\mathrm{mg}, 0.005 \mathrm{mmol}), \mathrm{Cs}_{2} \mathrm{CO}_{3}(130.3 \mathrm{mg}, 0.4 \mathrm{mmol})$ and $\mathbf{2 a}(51.4 \mathrm{mg}, 0.2 \mathrm{mmol})$ and flushed with nitrogen gas. 1-iodo-4-methoxybenzene (93.6 mg, $0.4 \mathrm{mmol})$ and dried DMF (2 mL) were added to the tube under nitrogen atmosphere. The sealed tube was heated at $90{ }^{\circ} \mathrm{C}$ for $15 \mathrm{~h}$ in a heating module with stirring. The mixture was cooled to r.t. and diluted with $\mathrm{H}_{2} \mathrm{O}$, the layers were separated, and the aqueous layer was extracted with EtOAc. The combined organic layers were washed with saturated brines, dried over $\mathrm{Na}_{2} \mathrm{SO}_{4}$, filtered, and concentrated by rotary evaporation. The crude mixture was purified by column chromatography on silica gel to afford the corresponding product 6 (38.9 $\mathrm{mg}$, $82 \%)$ as a yellow solid.

Compound 7 was prepared according to the procedures reported in the literature. ${ }^{[1]}$ An oven-dried Schlenk tube equipped with a stir-bar, $2 \mathbf{p}(0.2 \mathrm{mmol}, 48.6 \mathrm{mg})$ and $\mathrm{Cu}_{2} \mathrm{O}(0.08 \mathrm{mmol}, 11.6$ $\mathrm{mg}$ ) were combined. A balloon filled with $\mathrm{O}_{2}$ was connected to the Schlenk tube via the side tube and purged for 3 times, then TMSCN $(0.2 \mathrm{mmol}, 19.8 \mathrm{mg}), N, N, N^{\prime}, N^{\prime}$-tetramethyl-ethane-1,2-diamine $(0.24 \mathrm{mmol}, 27.8 \mathrm{mg}), \mathrm{Et}_{3} \mathrm{~N}(0.6 \mathrm{mmol}, 60.6 \mathrm{mg})$ and dry $\mathrm{CH}_{3} \mathrm{CN}(2 \mathrm{~mL})$ were injected via syringe. The mixture was stirred at $25{ }^{\circ} \mathrm{C}$ for 12 hours. The resultant mixture was evaporated under reduced pressure and the residue was directly purified by preparative thin-layer chromatography (PTLC; petroleum ether/ethyl acetate as the eluent) to afford $7(21.3 \mathrm{mg}, 75 \%)$ as light yellow solid.

Compound 8 was prepared according to the modified procedures reported in the literature. ${ }^{[10]} \mathrm{An}$ Schlenk tube equipped with a stir-bar, 2a (51.4 mg, $0.2 \mathrm{mmol}), \operatorname{PhSSPh}(24.0 \mathrm{mg}, 0.11 \mathrm{mmol}), \mathrm{CuCl}$ (4.0 mg, $0.04 \mathrm{mmol})$, dtbpy (14.4 mg, $0.054 \mathrm{mmol})$, DMSO $(0.3 \mathrm{~mL}), \mathrm{H}_{2} \mathrm{O}(0.15 \mathrm{~mL})$ were added to the tube under nitrogen atmosphere. The sealed tube was heated at $80^{\circ} \mathrm{C}$ for $10 \mathrm{~h}$ in a heating module with stirring. The mixture was cooled to r.t. and diluted with $\mathrm{H}_{2} \mathrm{O}(10 \mathrm{~mL})$, the layers were separated, and the aqueous layer was extracted with EtOAc. The combined organic layers were washed with saturated brines, dried over $\mathrm{Na}_{2} \mathrm{SO}_{4}$, filtered, and concentrated by rotary evaporation. The crude mixture was purified by column chromatography on silica gel to afford the corresponding product 8 (19.1 mg, 40\%) as a light yellow solid. 


\section{Control experiments}

a) $\mathrm{BH}_{3} \cdot \mathrm{L}$ as promoters in $\mathrm{C} 2-\mathrm{H}$ borylation of $\mathbf{1 a}$

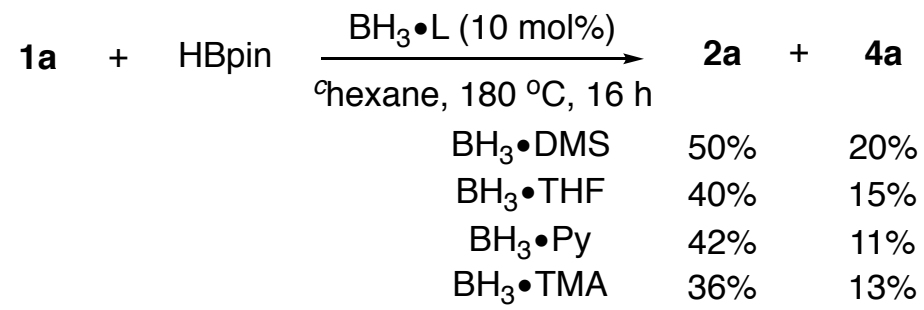

An oven-dried $25-\mathrm{mL}$ Schlenk tube equipped with a magnetic stirring bar was introduced inside a nitrogen-atmosphere glovebox. In the glovebox, the tube was charged with $\mathrm{BH}_{3} \cdot \mathrm{L}(0.04 \mathrm{mmol})$, sealed and then taken out of the glovebox. Then, 1a $(0.4 \mathrm{mmol}, 52.5 \mathrm{mg})$, HBpin $(2.4 \mathrm{mmol}, 307.2 \mathrm{mg})$ and 'hexane $(1.0 \mathrm{~mL})$ were added to the vessel under nitrogen atmosphere. The sealed tube was heated at $180{ }^{\circ} \mathrm{C}$ for $16 \mathrm{~h}$ in a heating module with stirring. After cooling the reaction mixture to room temperature, the solvent was evaporated under reduced pressure. The yields of $2 \mathbf{a}$ and $4 \mathbf{a}$ were determined by ${ }^{1} \mathrm{H}$ NMR using 3,4,5-trichloropyridine as internal standard.

b) Various simple nucleophiles as promoters

b) Various simple nucleophiles as promoters

\begin{tabular}{ccc|ccc}
$\mathbf{1 a}+$ HBpin & \multicolumn{5}{c}{ nucleophile (10 mol\%) } \\
\cline { 3 - 5 } & $\mathbf{c}$ chexane, $180^{\circ} \mathrm{C}, 16 \mathrm{~h}$ & $\mathbf{4}$ \\
\hline nucleophile & $\mathbf{2 a}$ & $\mathbf{4 a}$ & nucleophile & $\mathbf{2 a}$ & $\mathbf{4 a}$ \\
\hline $\mathrm{LiAlH}_{4}$ & $30 \%$ & $59 \%$ & $\mathrm{NaO}{ }^{\prime} \mathrm{Bu}$ & $42 \%$ & $3 \%$ \\
$\mathrm{LiO}^{t} \mathrm{Bu}$ & $48 \%$ & $11 \%$ & $\mathrm{KO}{ }^{t} \mathrm{Bu}$ & $46 \%$ & $14 \%$ \\
\hline
\end{tabular}

An oven-dried $25-\mathrm{mL}$ Schlenk tube equipped with a magnetic stirring bar was introduced inside a nitrogen-atmosphere glovebox. In the glovebox, the tube was charged with nucleophile promoter $(0.04$ $\mathrm{mmol})$, sealed and then taken out of the glovebox. Then, 1a $(0.4 \mathrm{mmol}, 52.5 \mathrm{mg})$, HBpin $(2.4 \mathrm{mmol}$, $307.2 \mathrm{mg})$ and ${ }^{c}$ hexane $(1.0 \mathrm{~mL})$ were added to the vessel under nitrogen atmosphere. The sealed tube was heated at $180^{\circ} \mathrm{C}$ for $16 \mathrm{~h}$ in a heating module with stirring. After cooling the reaction mixture to room temperature, the solvent was evaporated under reduced pressure. The yields of $\mathbf{2 a}$ and $4 \mathbf{a}$ were determined by ${ }^{1} \mathrm{H}$ NMR using 3,4,5-trichloropyridine as internal standard.

c) Inhibition of $\mathrm{C}-\mathrm{H}$ borylation of $\mathbf{1 a}$ by TMEDA

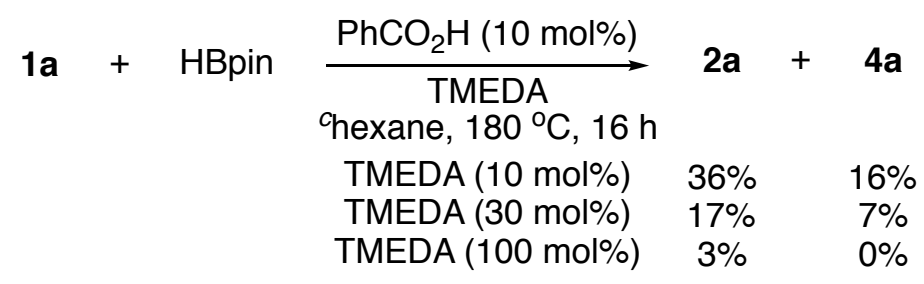


An oven-dried 25-mL Schlenk tube equipped with a magnetic stirring bar was charged with benzoic acid $(0.04 \mathrm{mmol}, 4.9 \mathrm{mg})$, and then flushed with nitrogen gas. 1a $(0.4 \mathrm{mmol}, 52.5 \mathrm{mg})$, HBpin (2.4 mmol, $307.2 \mathrm{mg}$ ), TMEDA (0.04 mmol, $4.6 \mathrm{mg}$; $0.12 \mathrm{mmol}, 13.9 \mathrm{mg} ; 0.4 \mathrm{mmol}, 46.5 \mathrm{mg}$ ) and chexane $(1.0 \mathrm{~mL})$ were added to the tube under nitrogen atmosphere. The sealed tube was heated at $180{ }^{\circ} \mathrm{C}$ for $16 \mathrm{~h}$ in a heating module with stirring. After cooling the reaction mixture to room temperature, the solvent was evaporated under reduced pressure. The yields of $\mathbf{2 a}$ and $4 \mathbf{a}$ were determined by ${ }^{1} \mathrm{H}$ NMR using 3,4,5-trichloropyridine as internal standard.

d) Stoichiometric reaction of $1 \mathbf{a}$ with $\mathrm{BH}_{3} \cdot \mathrm{DMS}$

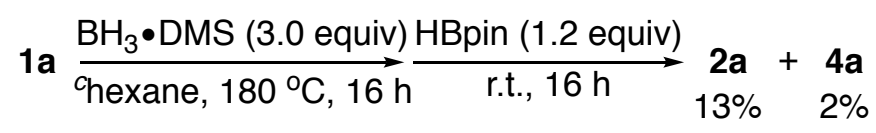

An oven-dried 25-mL Schlenk tube equipped with a magnetic stirring bar was introduced inside a nitrogen-atmosphere glovebox. In the glovebox, the tube was charged with $\mathrm{BH}_{3} \cdot \mathrm{DMS}(1.2 \mathrm{mmol}, 2.0$ M, 0.6 mL), sealed and then taken out of the glovebox. Then, $1 \mathrm{a}(0.4 \mathrm{mmol}, 52.5 \mathrm{mg})$ and ${ }^{c}$ hexane $(1.0 \mathrm{~mL})$ were added to the vessel under nitrogen atmosphere. The sealed tube was heated at $180{ }^{\circ} \mathrm{C}$ for $16 \mathrm{~h}$ in a heating module with stirring. After cooling the reaction mixture to room temperature, HBpin $(0.48 \mathrm{mmol}, 61.4 \mathrm{mg})$ was added to the tube under nitrogen atmosphere. After the reaction mixture was stirred at room temperature for $16 \mathrm{~h}$, the solvent was evaporated under reduced pressure. The yields of 2a and 4a were determined by ${ }^{1} \mathrm{H}$ NMR using 3,4,5-trichloropyridine as internal standard.

e) The stability of $\mathbf{3 a}$ in reaction system

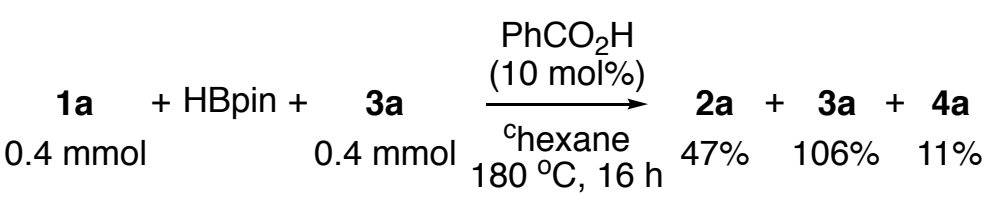

An oven-dried 25-mL Schlenk tube equipped with a magnetic stirring bar was charged with benzoic acid $(0.04 \mathrm{mmol}, 4.9 \mathrm{mg}), \mathbf{3 a}(0.4 \mathrm{mmol}, 102.9 \mathrm{mg})$, and then flushed with nitrogen gas. $1 \mathbf{a}(0.4 \mathrm{mmol}$, $52.5 \mathrm{mg})$, HBpin $(2.4 \mathrm{mmol}, 307.2 \mathrm{mg})$ and ${ }^{c}$ hexane $(1.0 \mathrm{~mL})$ were added to the tube under nitrogen atmosphere. The sealed tube was heated at $180^{\circ} \mathrm{C}$ for $16 \mathrm{~h}$ in a heating module with stirring. After cooling the reaction mixture to room temperature, the solvent was evaporated under reduced pressure. The yields of 2a, 3a and $\mathbf{4 a}$ were determined by ${ }^{1} \mathrm{H}$ NMR using 3,4,5-trichloropyridine as internal standard. 


\section{The ${ }^{11} B$ NMR analysis of the borylation reaction system}

Due to the high temperature and pressure of the reaction system, the ${ }^{11} \mathrm{~B}$ NMR analysis of the borylation reaction system was conducted by transferring the reaction mixture to NMR tube in glovebox. Several borylation reaction systems were conducted and sent for ${ }^{11} \mathrm{~B}$ NMR analysis (shown as bellow). However, unfortunately no other boron species except HBpin were observed from the spectra obtained.

Eq. 1: The reaction in cyclohexane was stopped, $\mathrm{CDCl}_{3}$ was added and sent for ${ }^{11} \mathrm{~B} \mathrm{NMR}$ analysis.

Eq. 2: The reaction in $\mathrm{D}_{12}$-cyclohexane was stopped and sent for ${ }^{11} \mathrm{~B}$ NMR analysis.

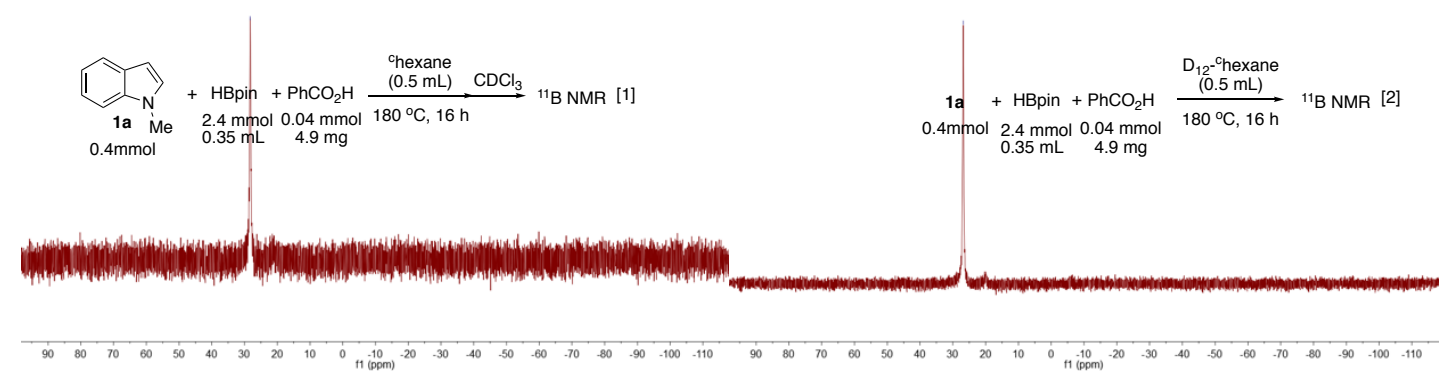

Eq. 3: The reaction in cyclohexane with the addition of dimethyl sulfide (DMS was added to form stable adduct with the possible $\mathrm{BH}_{3}$ species) was stopped, $\mathrm{CDCl}_{3}$ was added and sent for ${ }^{11} \mathrm{~B} \mathrm{NMR}$ analysis.

Eq. 4: The reaction in cyclohexane was stopped, dimethyl sulfide (DMS was added to form stable adduct with the possible $\mathrm{BH}_{3}$ species) and $\mathrm{CDCl}_{3}$ were added and sent for ${ }^{11} \mathrm{~B} \mathrm{NMR}$ analysis.

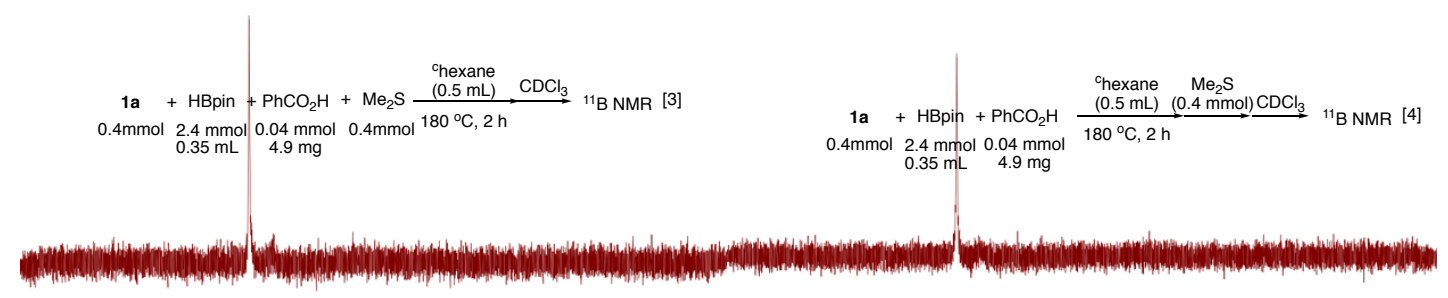

Eq. 5: The reaction with the addition of dimethyl sulfide (DMS was added to form stable adduct with the possible $\mathrm{BH}_{3}$ species) under neat conditions was stopped, $\mathrm{CDCl}_{3}$ was added and sent for ${ }^{11} \mathrm{~B} \mathrm{NMR}$ analysis.

Eq. 6: The reaction under neat conditions was stopped, dimethyl sulfide (DMS was added to form stable adduct with the possible $\mathrm{BH}_{3}$ species) and $\mathrm{CDCl}_{3}$ were added and sent for ${ }^{11} \mathrm{~B} \mathrm{NMR}$ analysis. 


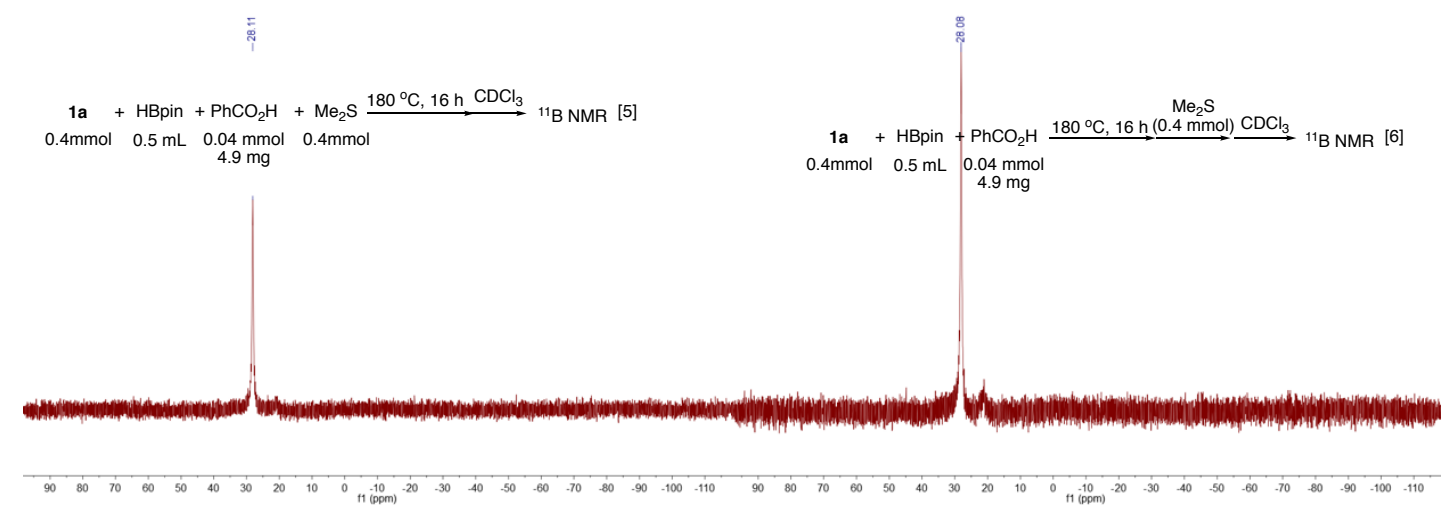

Eq. 7: The reaction in $\mathrm{D}_{12}$-cyclohexane was stopped, THF (THF was added to increase the solubility of the possible $\mathrm{BH}_{3}$ species) was added and sent for ${ }^{11} \mathrm{~B}$ NMR analysis.

Eq. 8: The reaction in cyclohexane was stopped, THF and $\mathrm{CDCl}_{3}$ was added and sent for ${ }^{11} \mathrm{~B}$ NMR analysis.

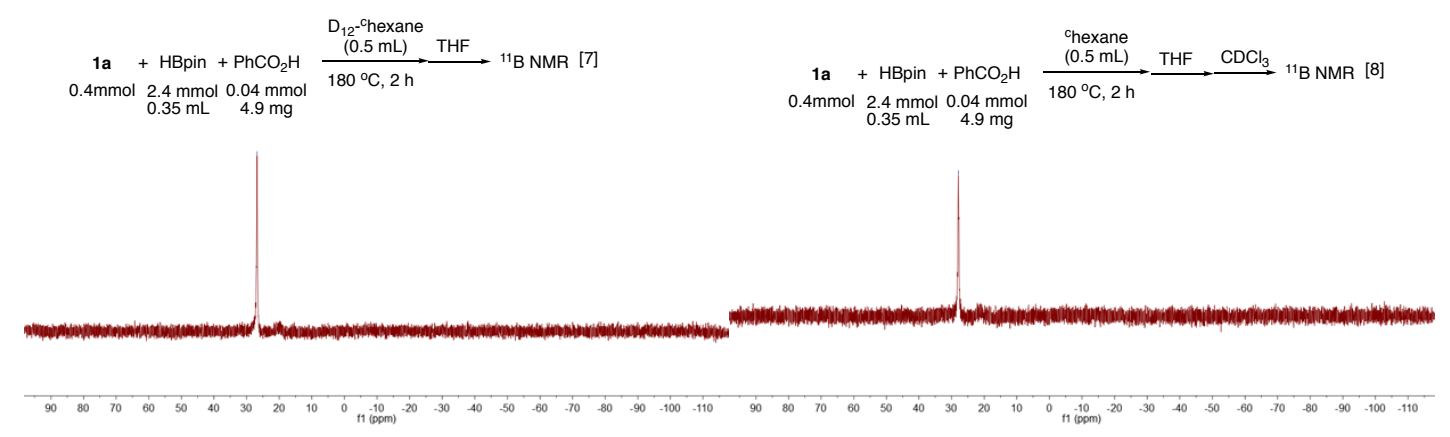




\section{Monitoring of the borylation of 1a with pinacolborane}

An oven-dried 25-mL Schlenk tube equipped with a magnetic stirring bar was charged with benzoic acid $(0.04 \mathrm{mmol}, 4.9 \mathrm{mg})$, and then flushed with nitrogen gas. 1a $(0.4 \mathrm{mmol}, 52.5 \mathrm{mg})$, HBpin (2.4 $\mathrm{mmol}, 307.2 \mathrm{mg})$ and ${ }^{c}$ hexane $(1.0 \mathrm{~mL})$ were added to the tube under nitrogen atmosphere. The sealed tube was heated at $180^{\circ} \mathrm{C}$ in a heating module with stirring for $0 \mathrm{~h}, 0.5 \mathrm{~h}, 1.0 \mathrm{~h}, 2.0 \mathrm{~h}, 3.0 \mathrm{~h}, 6.0 \mathrm{~h}, 9.0$ $\mathrm{h}, 12.0 \mathrm{~h}, 14.0 \mathrm{~h}, 16.0 \mathrm{~h}$ respectively. After cooling the reaction mixture to room temperature, the solvent was evaporated under reduced pressure. The yields of $\mathbf{2 a}, \mathbf{3 a}$ and $\mathbf{4 a}$ were determined by ${ }^{1} \mathrm{H}$ NMR using 3,4,5-trichloropyridine as internal standard. 


\section{Analytical data of products}

\section{1-Methyl-2-(4,4,5,5-tetramethyl-1,3,2-dioxaborolan-2-yl)-1H-indole(2a) ${ }^{[11]}$}<smiles>Cn1c(CBr)cc2ccccc21</smiles>

2a

White solid (72.0 mg, 70\%). Eluent: PE/EA (200/1). ${ }^{1} \mathrm{H}$ NMR $\left(400 \mathrm{MHz}, \mathrm{CDCl}_{3}\right) \delta 7.63(\mathrm{~d}, J=8.0 \mathrm{~Hz}$, 1H), 7.32 (d, $J=8.0 \mathrm{~Hz}, 1 \mathrm{H}), 7.23-7.26(\mathrm{~m}, 1 \mathrm{H}), 7.13$ (s, 1H), 7.07 (t, $J=8.0 \mathrm{~Hz}, 1 \mathrm{H}), 3.95$ (s, 3H), $1.34(\mathrm{~s}, 12 \mathrm{H}) ;{ }^{13} \mathrm{C}$ NMR $\left(100 \mathrm{MHz}, \mathrm{CDCl}_{3}\right) \delta 140.2,127.9,123.3,121.7,119.4,114.4,109.8,83.8$, $32.3,24.9$.

\section{1-Butyl-2-(4,4,5,5-tetramethyl-1,3,2-dioxaborolan-2-yl)-1H-indole(2b) ${ }^{[11]}$}<smiles>CC(C)(C)n1c(Br)cc2ccccc21</smiles>

2b

Light yellow oil (81.4 mg, 68\%). Eluent: PE/EA (200/1). ${ }^{1} \mathrm{H}$ NMR (400 MHz, $\left.\mathrm{CDCl}_{3}\right) \delta 7.63(\mathrm{~d}, J=$ $8.0 \mathrm{~Hz}, 1 \mathrm{H}), 7.36$ (d, $J=8.0 \mathrm{~Hz}, 1 \mathrm{H}), 7.25-7.21(\mathrm{~m}, 1 \mathrm{H}), 7.12(\mathrm{~s}, 1 \mathrm{H}), 7.08-7.04(\mathrm{~m}, 1 \mathrm{H}), 4.41$ (t, $J$ $=7.2 \mathrm{~Hz}, 2 \mathrm{H}), 1.78-1.71(\mathrm{~m}, 2 \mathrm{H}), 1.36(\mathrm{~s}, 12 \mathrm{H}), 1.33-1.29(\mathrm{~m}, 2 \mathrm{H}), 0.92(\mathrm{t}, J=7.6 \mathrm{~Hz}, 3 \mathrm{H}) ;{ }^{13} \mathrm{C} \mathrm{NMR}$ $\left(100 \mathrm{MHz}, \mathrm{CDCl}_{3}\right) \delta 139.3,128.0,123.0,121.7,119.1,114.4,109.9,83.6,45.2,33.1,24.8,20.2,13.9$.

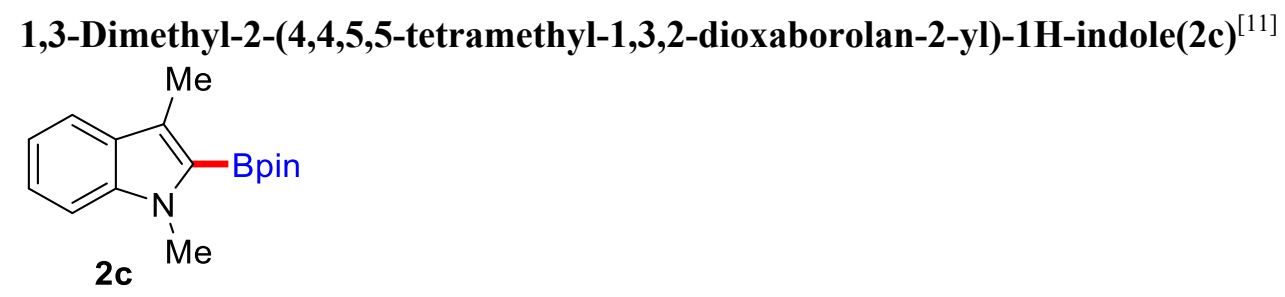

White solid (78.1 mg, 72\%). Eluent: PE/EA (200/1). ${ }^{1} \mathrm{H}$ NMR (400 MHz, $\left.\mathrm{CDCl}_{3}\right) \delta 7.61(\mathrm{~d}, J=8.0 \mathrm{~Hz}$, $1 \mathrm{H}), 7.22-7.28(\mathrm{~m}, 2 \mathrm{H}), 7.08-7.04(\mathrm{~m}, 1 \mathrm{H}), 3.91(\mathrm{~s}, 3 \mathrm{H}), 2.56(\mathrm{~s}, 3 \mathrm{H}), 1.35(\mathrm{~s}, 12 \mathrm{H}) ;{ }^{13} \mathrm{C}$ NMR $(100$ $\left.\mathrm{MHz}, \mathrm{CDCl}_{3}\right) \delta 139.9,128.5,124.8,123.4,119.8,118.5,109.5,83.3,32.2,25.0,10.3$.

\section{1,4-Dimethyl-2-(4,4,5,5-tetramethyl-1,3,2-dioxaborolan-2-yl)-1H-indole(2d $)^{[11]}$}<smiles>COc1cccc2c1cc(Cc1ccccc1)n2C</smiles>

White solid (81.4 mg, 75\%). Eluent: PE/EA (200/1). ${ }^{1} \mathrm{H}$ NMR (400 MHz, $\left.\mathrm{CDCl}_{3}\right) \delta 7.18-7.16(\mathrm{~m}, 3 \mathrm{H})$, 6.88-6.86 (m, 1H), $3.96(\mathrm{~s}, 3 \mathrm{H}), 2.55(\mathrm{~s}, 3 \mathrm{H}), 1.37(\mathrm{~s}, 12 \mathrm{H}) ;{ }^{13} \mathrm{C} \mathrm{NMR}\left(100 \mathrm{MHz}, \mathrm{CDCl}_{3}\right) \delta 140.1$, 131.3, 127.9, 123.5, 119.4, 112.9, 107.4, 83.7, 32.4, 24.9, 18.7. 


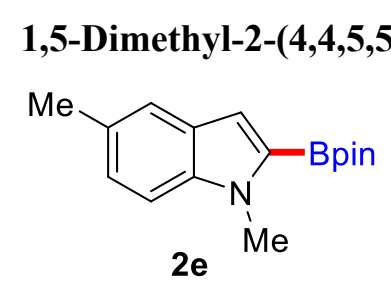

White solid (70.5 mg, 65\%). Eluent: PE/EA (200/1). ${ }^{1} \mathrm{H}$ NMR (400 MHz, $\left.\mathrm{CDCl}_{3}\right) \delta 7.41$ (s, 1H), 7.22 $(\mathrm{d}, J=8.0 \mathrm{~Hz}, 1 \mathrm{H}), 7.08(\mathrm{~d}, J=8.0 \mathrm{~Hz}, 1 \mathrm{H}), 7.03(\mathrm{~s}, 1 \mathrm{H}), 3.94(\mathrm{~s}, 3 \mathrm{H}), 2.43(\mathrm{~s}, 3 \mathrm{H}), 1.36(\mathrm{~s}, 12 \mathrm{H}) ;{ }^{13} \mathrm{C}$ NMR $\left(100 \mathrm{MHz}, \mathrm{CDCl}_{3}\right) \delta 138.8,128.5,128.1,125.1,121.0,113.7,109.4,83.7,32.3,24.9,21.4$.

\section{1,6-Dimethyl-2-(4,4,5,5-tetramethyl-1,3,2-dioxaborolan-2-yl)-1H-indole(2f $)^{[11]}$}<smiles>CCCCn1c(Cc2ccccc2)cc2ccc(C)cc21</smiles>

White solid (66.2 mg, 61\%). Eluent: PE/EA (200/1). ${ }^{1} \mathrm{H}$ NMR $\left(400 \mathrm{MHz}, \mathrm{CDCl}_{3}\right) \delta 7.51(\mathrm{~d}, J=8.0 \mathrm{~Hz}$, 1H), $7.13(\mathrm{~s}, 1 \mathrm{H}), 7.08(\mathrm{~s}, 1 \mathrm{H}), 6.92(\mathrm{~d}, J=8.0 \mathrm{~Hz}, 1 \mathrm{H}), 3.93(\mathrm{~s}, 3 \mathrm{H}), 2.49(\mathrm{~s}, 3 \mathrm{H}), 1.36(\mathrm{~s}, 12 \mathrm{H}) ;{ }^{13} \mathrm{C}$ NMR $\left(100 \mathrm{MHz}, \mathrm{CDCl}_{3}\right) \delta 140.7,133.2,125.8,121.4,121.2,114.2,109.6,83.6,32.2,24.9,22.1$.

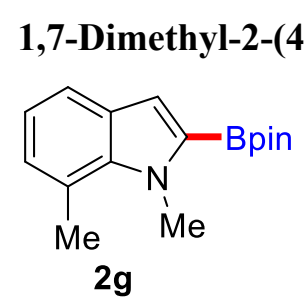

White solid (58.6 mg, 54\%). Eluent: PE/EA (200/1). ${ }^{1} \mathrm{H}$ NMR (400 MHz, $\left.\mathrm{CDCl}_{3}\right) \delta$ 7.46-7.43 (m, 1H), $7.09(\mathrm{~s}, 1 \mathrm{H}), 6.92-6.91(\mathrm{~m}, 2 \mathrm{H}), 4.26(\mathrm{~s}, 3 \mathrm{H}), 2.79(\mathrm{~s}, 3 \mathrm{H}), 1.35(\mathrm{~s}, 12 \mathrm{H}) ;{ }^{13} \mathrm{C}$ NMR $(100 \mathrm{MHz}$, $\left.\mathrm{CDCl}_{3}\right) \delta 139.2,128.9,126.0,121.7,119.9,119.5,115.1,83.7,35.7,24.8,20.5$.

\section{5-Methoxy-1-methyl-2-(4,4,5,5-tetramethyl-1,3,2-dioxaborolan-2-yl)-1H-indole(2h) ${ }^{[11]}$}<smiles>COc1ccc2c(c1)cc(Cc1ccccc1)n2C</smiles>

White solid (67.8 mg, 59\%). Eluent: PE/EA (100/1). ${ }^{1} \mathrm{H}$ NMR $\left(400 \mathrm{MHz}, \mathrm{CDCl}_{3}\right) \delta 7.23(\mathrm{~d}, J=9.2 \mathrm{~Hz}$, $1 \mathrm{H}), 7.05$ (d, $J=2.4 \mathrm{~Hz}, 1 \mathrm{H}), 7.03(\mathrm{~s}, 1 \mathrm{H}), 6.95-6.92(\mathrm{~m}, 1 \mathrm{H}), 3.94(\mathrm{~s}, 3 \mathrm{H}), 3.84(\mathrm{~s}, 3 \mathrm{H}), 1.36$ (s, 12H); ${ }^{13} \mathrm{C}$ NMR $\left(100 \mathrm{MHz}, \mathrm{CDCl}_{3}\right) \delta 154.0,135.9,128.0,114.4,113.5,110.5,102.1,83.7,55.8,32.4$, 24.9 . 


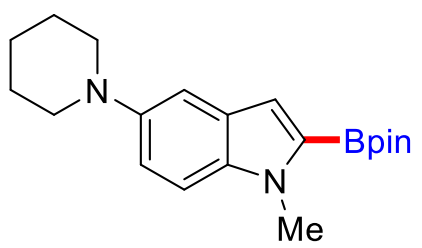

$2 \mathbf{i}$

White solid (61.2 mg, 45\%). Eluent: PE/EA (80/1). ${ }^{1} \mathrm{H}$ NMR (400 MHz, $\left.\mathrm{CDCl}_{3}\right) \delta 7.23(\mathrm{~d}, J=8.4 \mathrm{~Hz}$, 1H), $7.14(\mathrm{~s}, 1 \mathrm{H}), 7.09(\mathrm{~d}, J=8.8 \mathrm{~Hz}, 1 \mathrm{H}), 7.02(\mathrm{~s}, 1 \mathrm{H}), 3.92(\mathrm{~s}, 3 \mathrm{H}), 3.08(\mathrm{t}, J=5.2 \mathrm{~Hz}, 4 \mathrm{H})$, 1.79-1.74 (m, 4H), 1.59-1.53 (m, 2H), $1.35(\mathrm{~s}, 12 \mathrm{H}) ;{ }^{13} \mathrm{C} \mathrm{NMR}\left(100 \mathrm{MHz}, \mathrm{CDCl}_{3}\right) \delta 146.7,136.2$, 128.1, 118.5, 113.7, 110.0, 108.3, 83.6, 53.5, 32.3, 26.4, 24.8, 24.3. HRMS (ESI-TOF) m/z: $[\mathrm{M}+\mathrm{H}]^{+}$ Calcd for $\mathrm{C}_{20} \mathrm{H}_{30} \mathrm{BN}_{2} \mathrm{O}_{2}$ : 341.2395; Found 341.2407.

\section{5-Phenyl-1-methyl-2-(4,4,5,5-tetramethyl-1,3,2-dioxaborolan-2-yl)-1H-indole(2j) $)^{[11]}$}

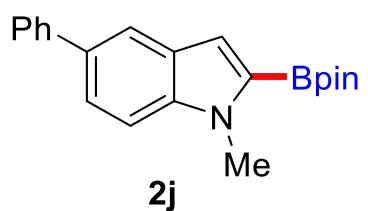

White solid (84.0 mg, 63\%). Eluent: PE/EA (80/1). ${ }^{1} \mathrm{H}$ NMR (400 MHz, $\left.\mathrm{CDCl}_{3}\right) \delta 7.85(\mathrm{~d}, J=1.6 \mathrm{~Hz}$, 1H), 7.67-7.65 (m, 2H), 7.54-7.52 (m, 1H), 7.45-7.39 (m, 3H), $7.30(\mathrm{t}, J=7.2 \mathrm{~Hz}, 1 \mathrm{H}), 7.17(\mathrm{~s}, 1 \mathrm{H})$, 3.99 (s, 3H), 1.37 (s, 12H); ${ }^{13} \mathrm{C}$ NMR $\left(100 \mathrm{MHz}, \mathrm{CDCl}_{3}\right) \delta 142.4,139.7,132.8,128.6,128.3,127.3$, $126.3,123.2,119.9,114.6,109.9,83.8,32.4,24.8$.

\section{1-Methyl-3-(4,4,5,5-tetramethyl-1,3,2-dioxaborolan-2-yl)-5-(trifluoromethyl)-1H-indole (2k)}<smiles>Cn1c(Cc2ccccc2)cc2cc(C(F)(F)F)ccc21</smiles>

White solid (85.8 mg, 66\%). Eluent: PE/EA (200/1). ${ }^{1} \mathrm{H}$ NMR (400 MHz, $\left.\mathrm{CDCl}_{3}\right) \delta 7.93(\mathrm{~s}, 1 \mathrm{H}), 7.47$ (d, $J=8.4 \mathrm{~Hz}, 1 \mathrm{H}), 7.39$ (d, $J=8.8 \mathrm{~Hz}, 1 \mathrm{H}), 7.19$ (s, 1H), 3.99 (s, 3H), 1.37 (s, 12H); ${ }^{13} \mathrm{C}$ NMR $(100$ $\left.\mathrm{MHz} \mathrm{CDCl}_{3}\right) \delta 141.1,129.5\left(\mathrm{C}-\mathrm{F},{ }^{1} J \mathrm{C}-\mathrm{F}=269.5 \mathrm{~Hz}\right), 127.0,126.8\left(\mathrm{C}-\mathrm{F},{ }^{1} J \mathrm{C}-\mathrm{F}=269.5 \mathrm{~Hz}\right), 124.1$ $\left(\mathrm{C}-\mathrm{F},{ }^{1} J \mathrm{C}-\mathrm{F}=269.5 \mathrm{~Hz}\right), 122.2\left(\mathrm{C}-\mathrm{F},{ }^{2} J \mathrm{C}-\mathrm{F}=31.5 \mathrm{~Hz}\right), 121.8\left(\mathrm{C}-\mathrm{F},{ }^{2} J \mathrm{C}-\mathrm{F}=31.5 \mathrm{~Hz}\right), 121.5(\mathrm{C}-\mathrm{F}$, $\left.{ }^{2} J \mathrm{C}-\mathrm{F}=31.5 \mathrm{~Hz}\right), 121.4\left(\mathrm{C}-\mathrm{F},{ }^{1} J \mathrm{C}-\mathrm{F}=269.5 \mathrm{~Hz}\right), 121.2\left(\mathrm{C}-\mathrm{F},{ }^{2} J \mathrm{C}-\mathrm{F}=31.5 \mathrm{~Hz}\right), 119.7\left(\mathrm{C}-\mathrm{F},{ }^{3} J \mathrm{C}-\mathrm{F}\right.$ $=3.0 \mathrm{~Hz}), 119.7\left(\mathrm{C}-\mathrm{F},{ }^{3} J \mathrm{C}-\mathrm{F}=3.0 \mathrm{~Hz}\right), 119.7\left(\mathrm{C}-\mathrm{F},{ }^{3} \mathrm{JC}-\mathrm{F}=3.0 \mathrm{~Hz}\right), 119.6\left(\mathrm{C}-\mathrm{F},{ }^{3} J \mathrm{C}-\mathrm{F}=3.0 \mathrm{~Hz}\right)$, $119.4\left(\mathrm{C}-\mathrm{F},{ }^{3} J \mathrm{C}-\mathrm{F}=4.5 \mathrm{~Hz}\right), 119.4\left(\mathrm{C}-\mathrm{F},{ }^{3} J \mathrm{C}-\mathrm{F}=4.5 \mathrm{~Hz}\right), 119.3\left(\mathrm{C}-\mathrm{F},{ }^{3} J \mathrm{C}-\mathrm{F}=4.5 \mathrm{~Hz}\right), 119.3(\mathrm{C}-\mathrm{F}$, $\left.{ }^{3} J \mathrm{C}-\mathrm{F}=4.5 \mathrm{~Hz}\right), 115.1,110.0,84.0,32.5,24.8$. HRMS (ESI-TOF) $\mathrm{m} / \mathrm{z}:[\mathrm{M}+\mathrm{H}]^{+}$Calcd for $\mathrm{C}_{16} \mathrm{H}_{20} \mathrm{BF}_{3} \mathrm{NO}_{2}$ 326.1534; Found 326.1524. 


\section{5-Fluoro-1-methyl-2-(4,4,5,5-tetramethyl-1,3,2-dioxaborolan-2-yl)-1H-indole(2I) ${ }^{[11]}$}<smiles>Cn1c(Cc2ccccc2)cc2cc(F)ccc21</smiles>

White solid (70.4 mg, 64\%). Eluent: PE/EA (200/1). ${ }^{1} \mathrm{H}$ NMR (400 MHz, $\left.\mathrm{CDCl}_{3}\right) \delta 7.27-7.22(\mathrm{~m}, 2 \mathrm{H})$, $7.06(\mathrm{~s}, 1 \mathrm{H}), 7.01\left(\mathrm{td}, J_{1}=9.2 \mathrm{~Hz}, J_{2}=2.4 \mathrm{~Hz}, 1 \mathrm{H}\right), 3.95(\mathrm{~s}, 3 \mathrm{H}), 1.36(\mathrm{~s}, 12 \mathrm{H}) ;{ }^{13} \mathrm{C} \mathrm{NMR}(100 \mathrm{MHz}$, $\left.\mathrm{CDCl}_{3}\right) \delta 158.9\left(\mathrm{C}-\mathrm{F},{ }^{1} \mathrm{JC}-\mathrm{F}=233.0 \mathrm{~Hz}\right), 156.6\left(\mathrm{C}-\mathrm{F},{ }^{1} \mathrm{JC}-\mathrm{F}=233.0 \mathrm{~Hz}\right), 136.9,127.9\left(\mathrm{C}-\mathrm{F},{ }^{3} J \mathrm{C}-\mathrm{F}\right.$ $=10.0 \mathrm{~Hz}), 127.8\left(\mathrm{C}-\mathrm{F},{ }^{3} J \mathrm{C}-\mathrm{F}=10.0 \mathrm{~Hz}\right), 113.8\left(\mathrm{C}-\mathrm{F},{ }^{4} J \mathrm{C}-\mathrm{F}=5.0 \mathrm{~Hz}\right), 113.8\left(\mathrm{C}-\mathrm{F},{ }^{4} J \mathrm{C}-\mathrm{F}=5.0 \mathrm{~Hz}\right)$, $112.1\left(\mathrm{C}-\mathrm{F},{ }^{2} J \mathrm{C}-\mathrm{F}=26.0 \mathrm{~Hz}\right), 111.8\left(\mathrm{C}-\mathrm{F},{ }^{2} J \mathrm{C}-\mathrm{F}=26.0 \mathrm{~Hz}\right), 110.4\left(\mathrm{C}-\mathrm{F},{ }^{3} J \mathrm{C}-\mathrm{F}=10.0 \mathrm{~Hz}\right), 110.3$ $\left(\mathrm{C}-\mathrm{F},{ }^{3} J \mathrm{C}-\mathrm{F}=10.0 \mathrm{~Hz}\right), 105.8\left(\mathrm{C}-\mathrm{F},{ }^{2} J \mathrm{C}-\mathrm{F}=23.0 \mathrm{~Hz}\right), 105.6\left(\mathrm{C}-\mathrm{F},{ }^{2} J \mathrm{C}-\mathrm{F}=23.0 \mathrm{~Hz}\right), 83.9,32.5$, 24.8 .

\section{5-Chloro-1-methyl-2-(4,4,5,5-tetramethyl-1,3,2-dioxaborolan-2-yl)-1H-indole(2m $)^{[11]}$}<smiles>Cn1c(CBr)cc2cc(Cl)ccc21</smiles>

White solid (83.8 mg, 72\%). Eluent: PE/EA (200/1). ${ }^{1} \mathrm{H}$ NMR (400 MHz, $\left.\mathrm{CDCl}_{3}\right) \delta 7.60(\mathrm{~d}, J=8.0 \mathrm{~Hz}$, 1H), 7.26 (s, 1H), 7.21-7.18 (m, 1H), 7.05 (s, 1H), 3.95 (s, 3H), 1.37 (s, 12H); ${ }^{13} \mathrm{C}$ NMR (100 MHz, $\left.\mathrm{CDCl}_{3}\right) \delta 138.5,128.7,125.0,123.5,120.7,113.5,110.7,83.9,32.4,24.8$.

\section{5-Broime-1-methyl-2-(4,4,5,5-tetramethyl-1,3,2-dioxaborolan-2-yl)-1H-indole(2n ${ }^{[11]}$}

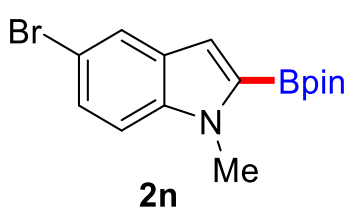

White solid (95.2 mg, 71\%). Eluent: PE/EA (200/1). ${ }^{1} \mathrm{H}$ NMR (400 MHz, $\left.\mathrm{CDCl}_{3}\right) \delta 7.76$ (s, 1H), 7.32 $(\mathrm{d}, J=8.8 \mathrm{~Hz}, 1 \mathrm{H}), 7.21(\mathrm{~d}, J=8.8 \mathrm{~Hz}, 1 \mathrm{H}), 7.04(\mathrm{~s}, 1 \mathrm{H}), 3.94(\mathrm{~s}, 3 \mathrm{H}), 1.36(\mathrm{~s}, 12 \mathrm{H}) ;{ }^{13} \mathrm{C}$ NMR $(100$ $\left.\mathrm{MHz}, \mathrm{CDCl}_{3}\right) \delta 138.8,129.4,126.0,123.9,113.5,112.7,111.2,83.9,32.4,24.9$.

\section{1-Methyl-2,5-bis(4,4,5,5-tetramethyl-1,3,2-dioxaborolan-2-yl)-1H-indole(2o)}

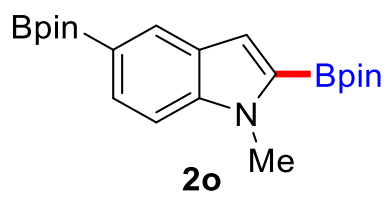

White solid (62.9 mg, 41\%). Eluent: PE/EA (80/1). ${ }^{1} \mathrm{H}$ NMR (400 MHz, $\left.\mathrm{CDCl}_{3}\right) \delta 8.16(\mathrm{~s}, 1 \mathrm{H}), 7.69$ (d, $J=8.4 \mathrm{~Hz}, 1 \mathrm{H}), 7.33$ (d, $J=8.4 \mathrm{~Hz}, 1 \mathrm{H}), 7.15(\mathrm{~s}, 1 \mathrm{H}), 3.96(\mathrm{~s}, 3 \mathrm{H}), 1.37$ (s, 24H); ${ }^{13} \mathrm{C}$ NMR $(100$ 
$\left.\mathrm{MHz}, \mathrm{CDCl}_{3}\right) \delta 142.0,129.8,129.1,127.6,115.1,109.1,83.7,83.4,32.3,24.9,24.8$. HRMS (ESI-TOF) m/z: $[\mathrm{M}+\mathrm{H}]^{+}$Calcd for $\mathrm{C}_{21} \mathrm{H}_{32} \mathrm{~B}_{2} \mathrm{NO}_{4}$ 384.2512; Found 384.2526.

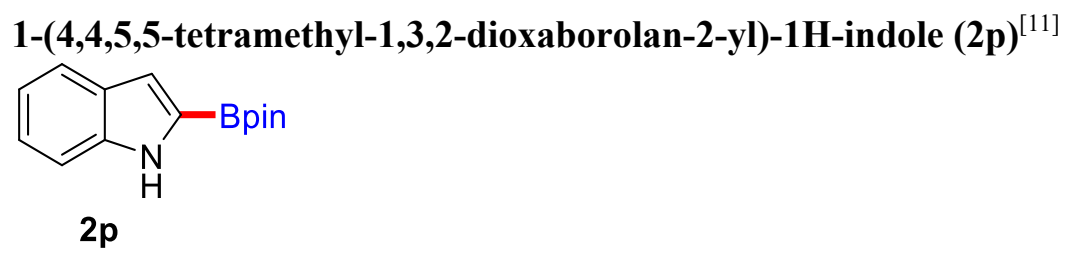

Light yellow solid (44.7 mg, 46\%). Eluent: PE/EA (50/1). ${ }^{1} \mathrm{H}$ NMR (400 MHz, $\left.\mathrm{CDCl}_{3}\right) \delta 8.74(\mathrm{~s}, 1 \mathrm{H})$, $7.66(\mathrm{~d}, J=8.0 \mathrm{~Hz}, 1 \mathrm{H}), 7.32(\mathrm{~d}, J=8.0 \mathrm{~Hz}, 1 \mathrm{H}), 7.22-7.18(\mathrm{~m}, 1 \mathrm{H}), 7.13-7.05(\mathrm{~m}, 2 \mathrm{H}), 1.32$ (s, $12 \mathrm{H}) ;{ }^{13} \mathrm{C} \mathrm{NMR}\left(100 \mathrm{MHz}, \mathrm{CDCl}_{3}\right) \delta 138.4,128.4,123.8,121.7,119.9,114.1,111.5,84.3,24.9$.

\section{1-Methyl-2-(4,4,5,5-tetramethyl-1,3,2-dioxaborolan-2-yl)-7-aza-1H-indole(2q $)^{[11]}$}<smiles>Cn1c(Cc2ccccc2)cc2cccnc21</smiles>

White solid (68.2 mg, 66\%). Eluent: PE/EA (200/1). ${ }^{1} \mathrm{H}$ NMR (400 MHz, $\left.\mathrm{CDCl}_{3}\right) \delta 8.40(\mathrm{~d}, J=4.4 \mathrm{~Hz}$, $1 \mathrm{H}), 7.92(\mathrm{~d}, J=8.0 \mathrm{~Hz}, 1 \mathrm{H}), 7.07(\mathrm{~s}, 1 \mathrm{H}), 7.04-7.01(\mathrm{~m}, 1 \mathrm{H}), 4.07(\mathrm{~s}, 3 \mathrm{H}), 1.37(\mathrm{~s}, 12 \mathrm{H}) ;{ }^{13} \mathrm{C} \mathrm{NMR}$ $\left(100 \mathrm{MHz}, \mathrm{CDCl}_{3}\right) \delta 150.3,144.8,129.6,120.0,115.5,112.1,83.9,30.9,24.8$.

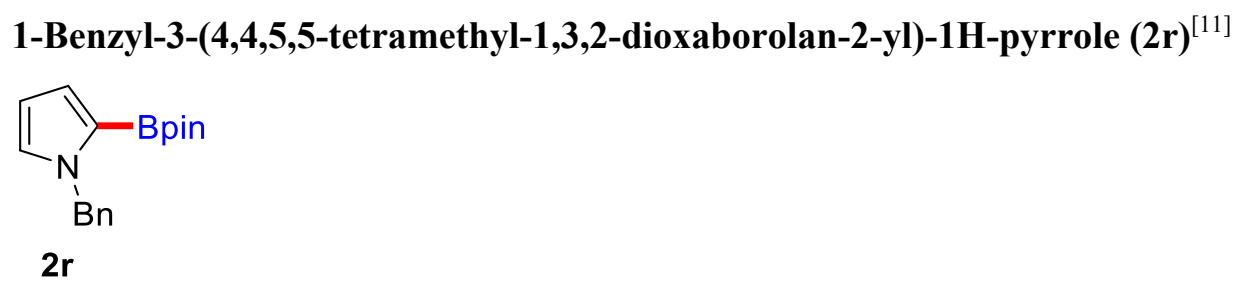

Light yellow solid (47.6 mg, 42\%). Eluent: PE/EA (300/1). ${ }^{1} \mathrm{H}$ NMR (400 MHz, $\left.\mathrm{CDCl}_{3}\right) \delta 7.28-7.24$ (m, 2H), 7.23-7.19 (m, 1H), $7.08(\mathrm{~d}, J=6.9 \mathrm{~Hz}, 2 \mathrm{H}), 6.90-6.88(\mathrm{~m}, 1 \mathrm{H}), 6.87-6.85(\mathrm{~m}, 1 \mathrm{H})$, 6.22-6.20 (m, 1H), $5.38(\mathrm{~s}, 2 \mathrm{H}), 1.22(\mathrm{~s}, 12 \mathrm{H}) ;{ }^{13} \mathrm{C} \mathrm{NMR}\left(100 \mathrm{MHz}, \mathrm{CDCl}_{3}\right) \delta 139.7,128.4,127.7$, $127.1,126.9,122.2,109.1,83.2,52.8,24.7$.

\section{1-methylindolin-2-one (5) ${ }^{[12]}$}<smiles>CN1C(=O)Cc2ccccc21</smiles> 
White solid (23.8 mg, 81\%). Eluent: PE/EA (60/1). ${ }^{1} \mathrm{H}$ NMR (400 MHz, $\left.\mathrm{CDCl}_{3}\right) \delta$ 7.30-7.22 (m, 2H), $7.04\left(\mathrm{t}, J=7.2 \mathrm{~Hz}, 6.81(\mathrm{~d}, J=8.0 \mathrm{~Hz}, 1 \mathrm{H}), 3.50(\mathrm{~s}, 2 \mathrm{H}), 3.20(\mathrm{~s}, 3 \mathrm{H}) ;{ }^{13} \mathrm{C} \mathrm{NMR}\left(100 \mathrm{MHz}, \mathrm{CDCl}_{3}\right) \delta\right.$ $175.1,145.1,127.8,124.5,124.3,122.3,108.0,35.7,26.1$.

\section{2-(4-methoxyphenyl)-1-methyl-1H-indole (6) ${ }^{[13]}$}<smiles>COc1ccc(-c2cc3ccccc3n2C)cc1</smiles>

Light yellow solid (38.9 mg, 82\%). Eluent: PE/EA (100/1). ${ }^{1} \mathrm{H}$ NMR (400 MHz, $\left.\mathrm{CDCl}_{3}\right) \delta 7.72(\mathrm{~d}, J=$ $8.0 \mathrm{~Hz}, 1 \mathrm{H}), 7.51(\mathrm{~d}, J=8.8 \mathrm{~Hz}, 2 \mathrm{H}), 7.42(\mathrm{~d}, J=8.0 \mathrm{~Hz}, 1 \mathrm{H}), 7.33(\mathrm{t}, J=6.8 \mathrm{~Hz}, 1 \mathrm{H}), 7.24(\mathrm{t}, J=6.8$ $\mathrm{Hz}, 1 \mathrm{H}), 7.07(\mathrm{~d}, J=8.8 \mathrm{~Hz}, 2 \mathrm{H}), 6.60(\mathrm{~s}, 1 \mathrm{H}), 3.92(\mathrm{~s}, 3 \mathrm{H}), 3.78(\mathrm{~s}, 3 \mathrm{H}) ;{ }^{13} \mathrm{C} \mathrm{NMR}\left(100 \mathrm{MHz}, \mathrm{CDCl}_{3}\right)$ $\delta 159.5,141.5,138.2,130.7,128.1,125.3,121.5,120.4,119.9,114.1,109.6,101.1,55.4,31.1$.

\section{H-indole-2-carbonitrile (7) ${ }^{[14]}$}<smiles>N#Cc1cc2ccccc2[nH]1</smiles>

Light yellow solid (21.3 mg, 75\%). Eluent: PE/EA (40/1). ${ }^{1} \mathrm{H}$ NMR (400 MHz, $\left.\mathrm{CDCl}_{3}\right) \delta 8.84(\mathrm{~s}, 1 \mathrm{H})$, $7.59(\mathrm{~d}, J=8.0 \mathrm{~Hz}, 1 \mathrm{H}), 7.35-7.28(\mathrm{~m}, 2 \mathrm{H}), 7.17-7.12(\mathrm{~m}, 2 \mathrm{H}) ;{ }^{13} \mathrm{C} \mathrm{NMR}\left(100 \mathrm{MHz}, \mathrm{CDCl}_{3}\right) \delta 136.9$, $126.3,126.2,122.1,121.7,114.45,111.8,106.1$.

\section{1-methyl-2-(phenylthio)-1H-indole (8) ${ }^{[15]}$}<smiles>Cn1c(-c2ccccc2)cc2ccccc21</smiles>

Yellow solid (19.1 mg, 40\%). Eluent: PE/EA (100/1). ${ }^{1} \mathrm{H}$ NMR (400 MHz, $\left.\mathrm{CDCl}_{3}\right) \delta 7.63(\mathrm{~d}, J=8.0$ $\mathrm{Hz}, 1 \mathrm{H}), 7.31-7.25$ (m, 2H), 7.21-7.08 (m, 4H), 7.04 (d, J=7.2 Hz, 2H), 6.94 (s, 1H), $3.66(\mathrm{~s}, 3 \mathrm{H}) ;{ }^{13} \mathrm{C}$ NMR $\left(100 \mathrm{MHz}, \mathrm{CDCl}_{3}\right) \delta 138.7,137.1,129.2,127.3,127.1,126.6,125.8,123.0,120.9,120.0,111.8$, $109.9,77.4,77.1,76.8,29.9$. 


\section{Reference}

[1] Fortes, M. P.; Bassaco, M. M.; Kaufman, T. S.; Silveira, C. C. RSC. Adv. 2014, 4, 34519.

[2] Huo, H.; Fu, C.; Harms, K.; Meggers, E. J. Am. Chem. Soc. 2014, 136, 2990.

[3] Zhang, Q. B.; Ban, Y. L.; Yuan, P. F.; Wu, L. Z.; Liu, Q. Green Chem. 2017, 19, 5559.

[4] Ji, Y. Z.; Li, H. J.; Wang, Y. R.; Zhang, Z. Y.; Wu, Y. C. Adv. Synth. Catal. 2020, 362, 1039.

[5] Ramgren, S. D.; Hie, L.; Ye, Y. X.; Garg, N. K. Org. Lett. 2013, 15, 3950.

[6] Sergeev, A. G.; Aritamkina, G. A.; Velezheva, V. S.; Fedorova, I. N.; Beleskaya, I. P. Russ. J. Org. Chem. 2005, 41, 860 .

[7] Prieto, M.; Zurita, E.; Munoz, L.; Williams, P. L.; Girat, E. J. Org. Chem. 2004, 69, 6812.

[8] Romero, E. A.; Peltier, J. L.; Jazzar, R.; Bertrand, G. Chem. Commun. 2016, 52, 10563.

[9] Smith, J. R.; Collins, B. S. L.; Hesse, M. J.; Graham, M. A.; Myers, E. L.; Aggarwal. V. K. J. Am. Chem. Soc. 2017, 139, 9148 .

[10] Dai, P. F.; Ning, X. S.; Wang, H.; Cui, X, C. ; Liu, J.; Qu, J. P.; Kang, Y. B. Angew. Chem. Int. Ed. 2019, $58,5392$.

[11] Zhong, Q.; Qin, S. X.; Yin, Y. Z.; Hu, J. J.; Zhang, H. Angew. Chem. Int. Ed. 2018, 57, 14891.

[12] Ghosh, S.; Chaudhuri, S.; Bisai, A. Org. Lett. 2015, 17, 1373.

[13] Gemoets, H. P. L.; Kalvet, I.; Nyuchev, A. V.; Erdmann, N.; Hessel, V.; Schoenebeck, F.; Noël, T. Chem. Sci. 2017, 8, 1046-1055.

[14] Xua, S. G.; Xiaomei Huang, X. M.; Hong, X. H.; Xu, B. Org. Lett. 2012, 14, 4614.

[15] Hostier,T.; Ferey, V.; Ricci, G.; Pardo, D. G.; Cossy, J. Chem. Commun. 2015, 51, 13898. 
10. NMR spectra of products

${ }^{1} \mathrm{H}$ NMR spectrum of $\mathbf{2 a}$ in $\mathrm{CDCl}_{3}(400 \mathrm{MHz})$

\begin{tabular}{|c|c|}
\hline 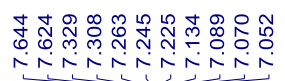 & זू. \\
\hline
\end{tabular}<smiles>Cn1c(Br)cc2ccccc21</smiles>

$2 a$

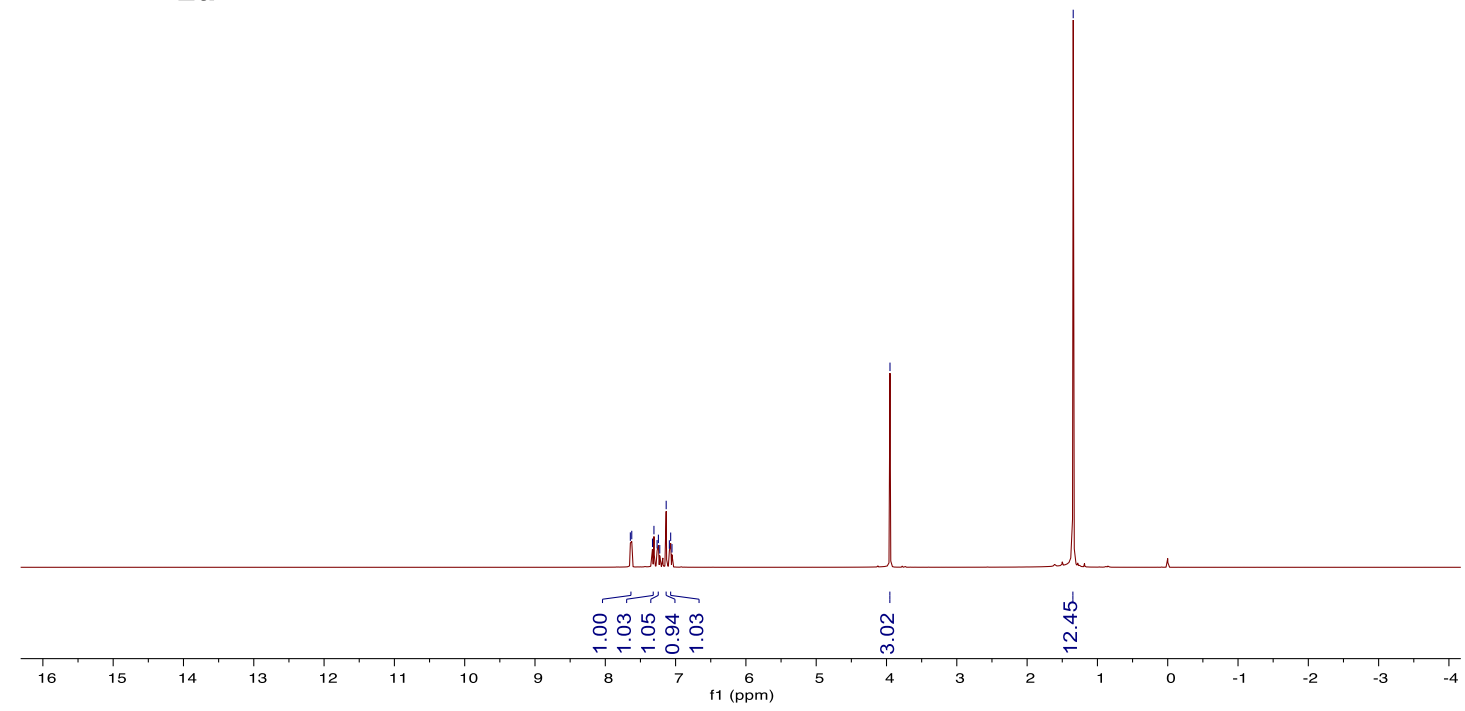

${ }^{13} \mathrm{C}$ NMR spectrum of $\mathbf{2 a}$ in $\mathrm{CDCl}_{3}(100 \mathrm{MHz})$

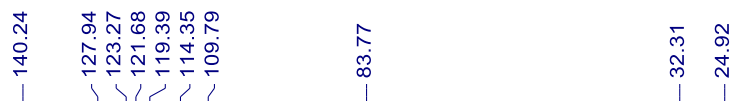<smiles>Cn1c(CBr)cc2ccccc21</smiles>

$2 a$

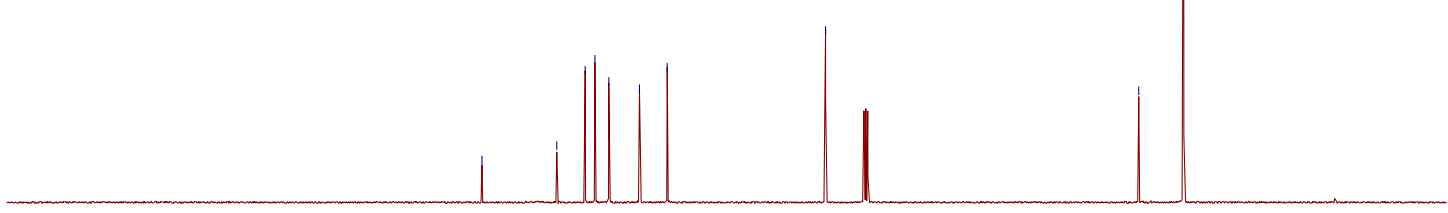

$210 \quad 200$

$\begin{array}{llll}190 & 180 & 170 & 160\end{array}$

$150 \quad 140 \quad 130$ 
${ }^{1} \mathrm{H}$ NMR spectrum of $\mathbf{2 c}$ in $\mathrm{CDCl}_{3}(400 \mathrm{MHz})$

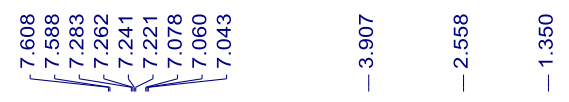<smiles>Cc1c(CBr)c2ccccc2n1C</smiles><smiles>Cc1c(CCc2ccccc2)n(C)c2ccccc12</smiles>

${ }^{13} \mathrm{C}$ NMR spectrum of $2 \mathrm{c}$ in $\mathrm{CDCl}_{3}(100 \mathrm{MHz})$

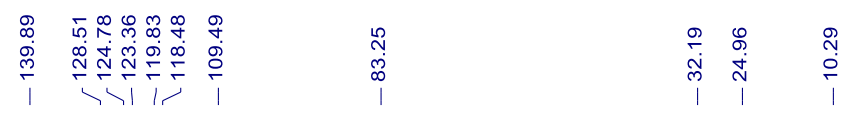

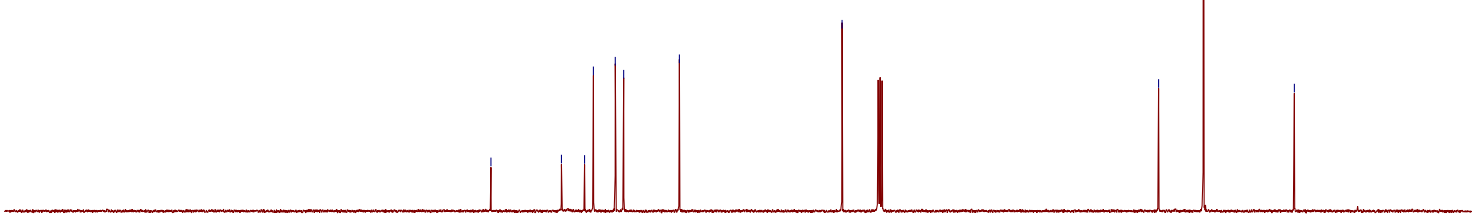

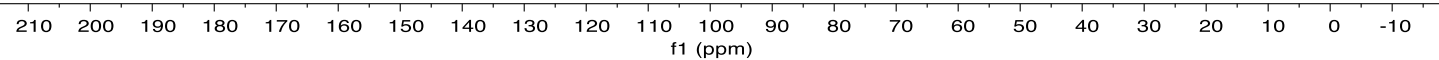


${ }^{1} \mathrm{H}$ NMR spectrum of $\mathbf{2 d}$ in $\mathrm{CDCl}_{3}(400 \mathrm{MHz})$

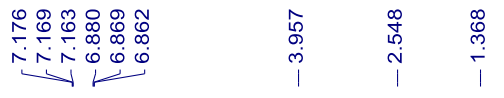<smiles>COc1cccc2c1cc(Cc1ccccc1)n2C</smiles>

2d

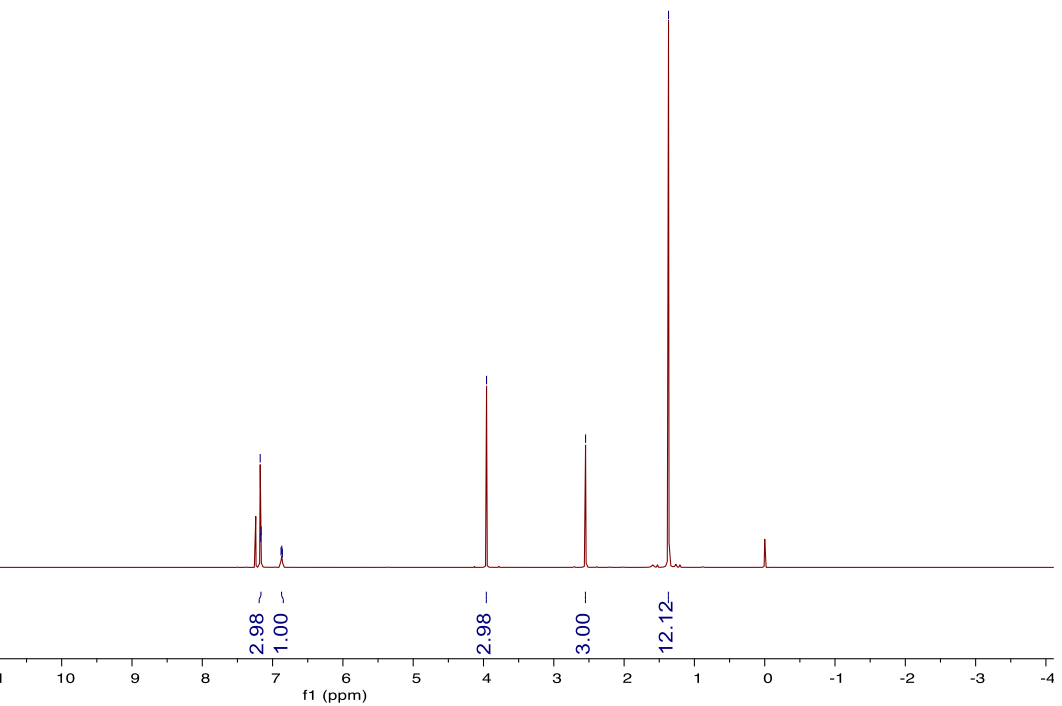

${ }^{13} \mathrm{C}$ NMR spectrum of $\mathbf{2 d}$ in $\mathrm{CDCl}_{3}(100 \mathrm{MHz})$

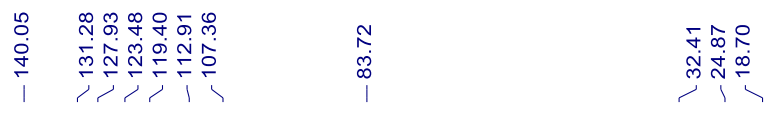<smiles>COc1cccc2c1cc(Cc1ccccc1)n2C</smiles> 
${ }^{1} \mathrm{H}$ NMR spectrum of $2 \mathbf{e}$ in $\mathrm{CDCl}_{3}(400 \mathrm{MHz})$

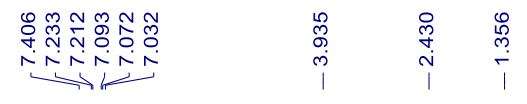<smiles>Cc1ccc2c(c1)cc(Cc1ccccc1)n2C</smiles>

$2 \mathrm{e}$

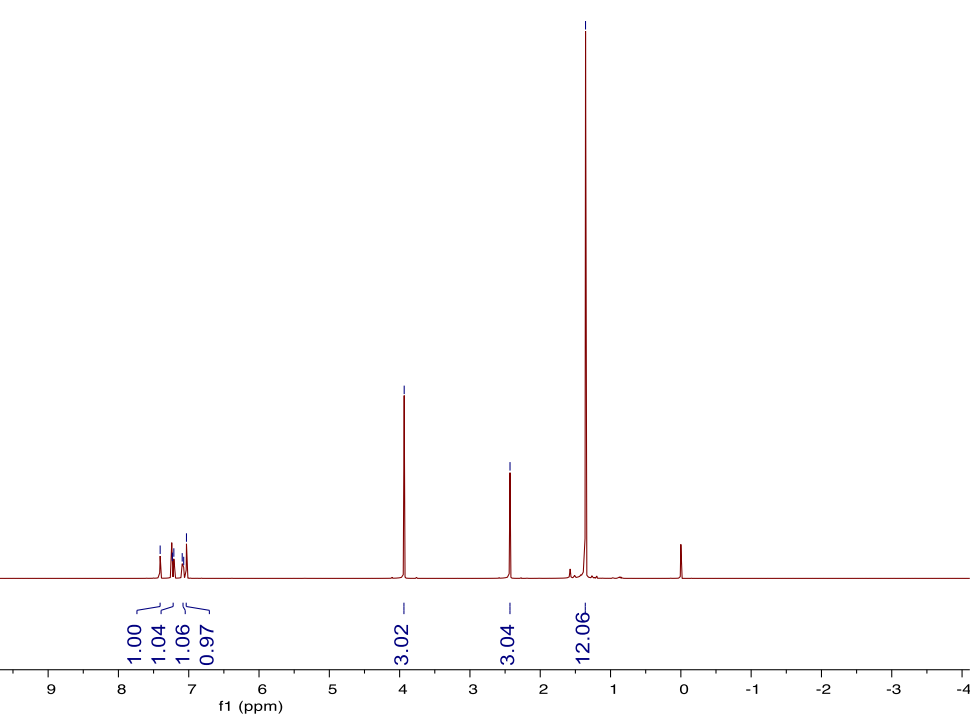

${ }^{13} \mathrm{C}$ NMR spectrum of $2 \mathrm{e}$ in $\mathrm{CDCl}_{3}(100 \mathrm{MHz})$

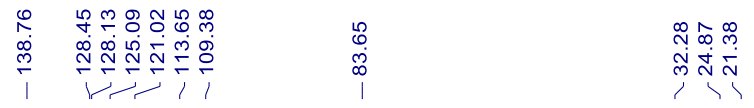<smiles>Cc1ccc2c(c1)cc(Cc1ccccc1)n2C</smiles>

$2 \mathrm{e}$

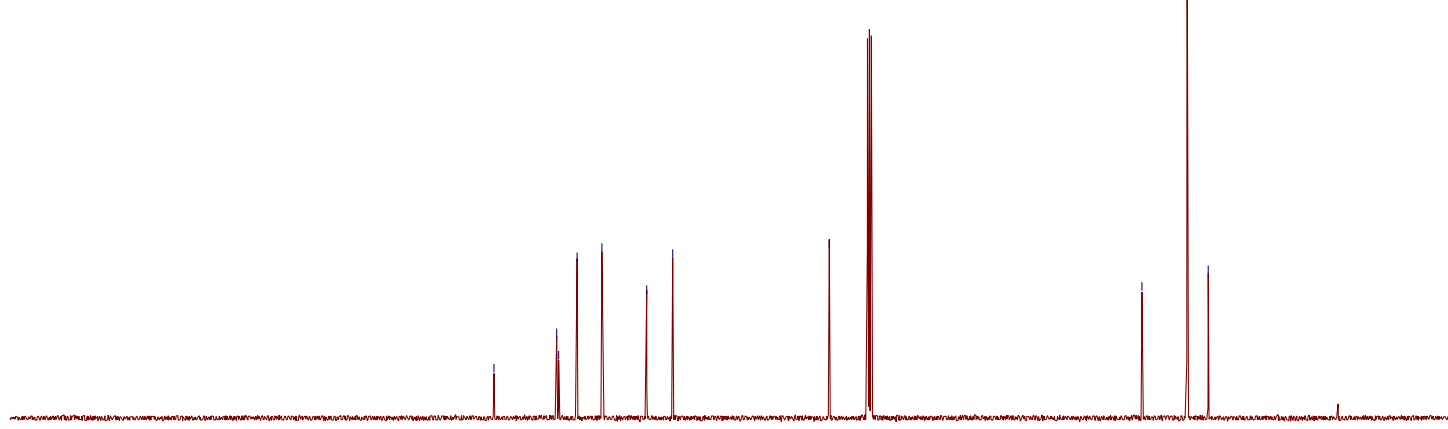

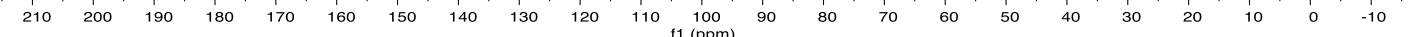


${ }^{1} \mathrm{H}$ NMR spectrum of $2 \mathbf{f}$ in $\mathrm{CDCl}_{3}(400 \mathrm{MHz})$

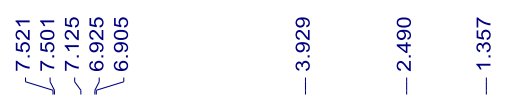

$\overbrace{M e}$ Bpin

$2 f$

${ }^{13} \mathrm{C}$ NMR spectrum of $\mathbf{2} \mathbf{f}$ in $\mathrm{CDCl}_{3}(100 \mathrm{MHz})$

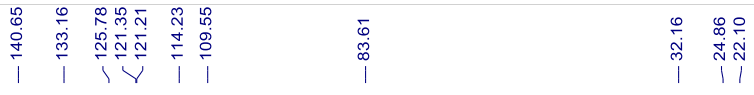

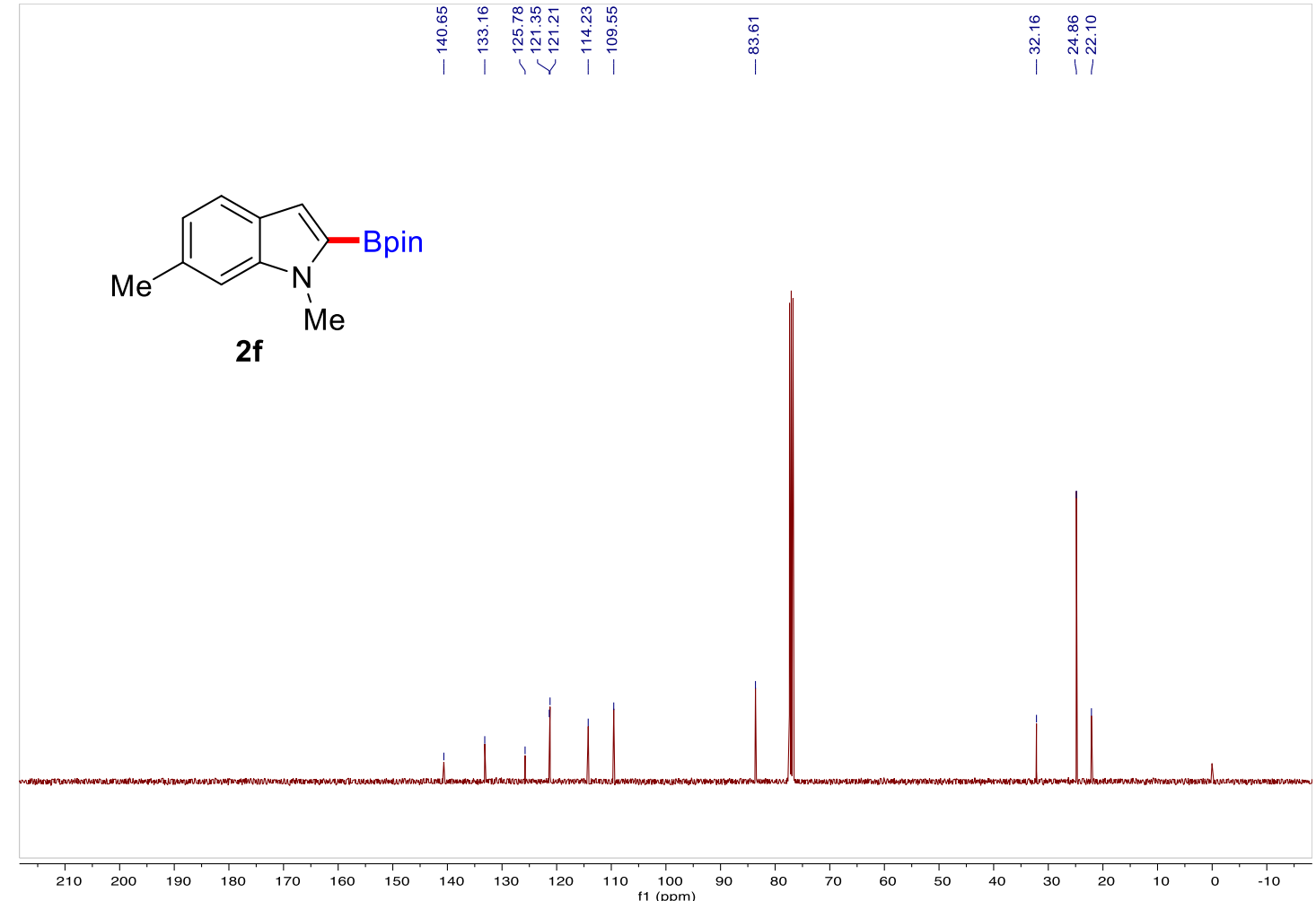

$2 f$
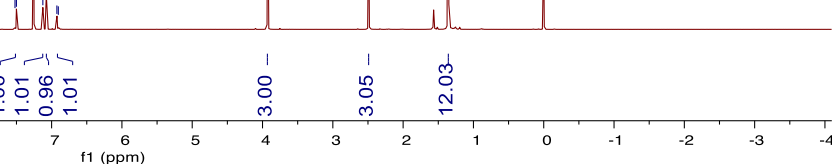
${ }^{1} \mathrm{H}$ NMR spectrum of $2 \mathrm{~g}$ in $\mathrm{CDCl}_{3}(400 \mathrm{MHz})$

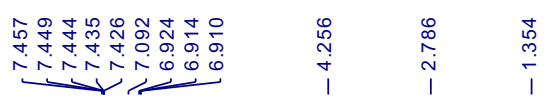<smiles>Cc1cccc2cc(Cc3ccccc3)n(C)c12</smiles>

$2 \mathrm{~g}$

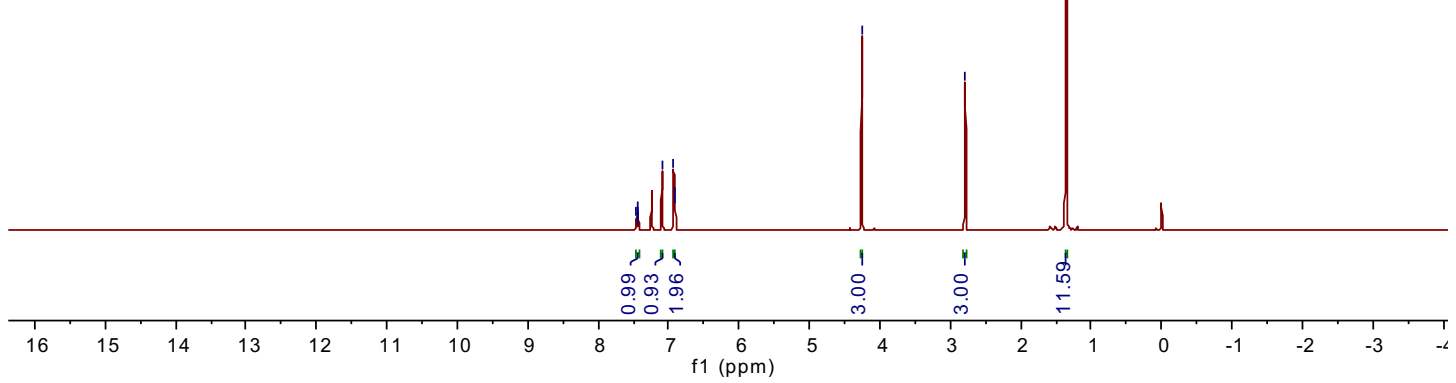

${ }^{13} \mathrm{C}$ NMR spectrum of $\mathbf{2 g}$ in $\mathrm{CDCl}_{3}(100 \mathrm{MHz})$

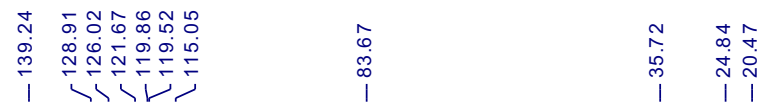<smiles>Cc1cccc2cc(CCc3ccccc3)n(C)c12</smiles>

$2 \mathrm{~g}$

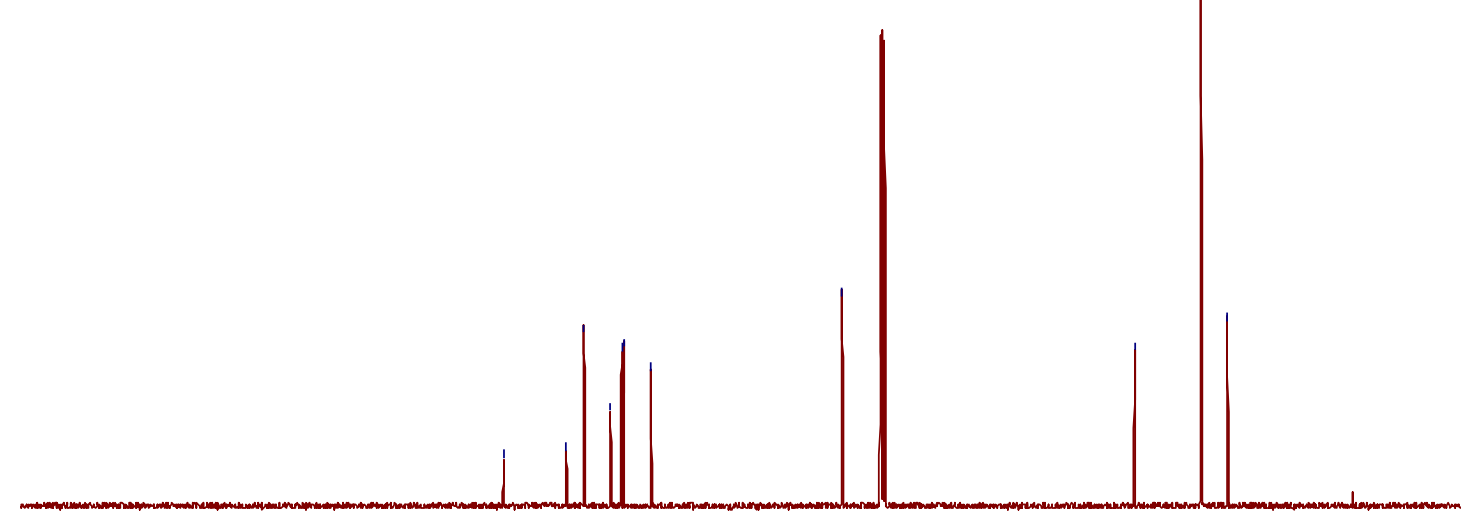

$\begin{array}{lllllllllllllllllllllllllll}210 & 200 & 190 & 180 & 170 & 160 & 150 & 140 & 130 & 120 & 110 & 100 & 90 & 80 & 70 & 60 & 50 & 40 & 30 & 20 & 10 & 0 & -10\end{array}$ 
${ }^{1} \mathrm{H}$ NMR spectrum of $\mathbf{2} \mathbf{h}$ in $\mathrm{CDCl}_{3}(400 \mathrm{MHz})$
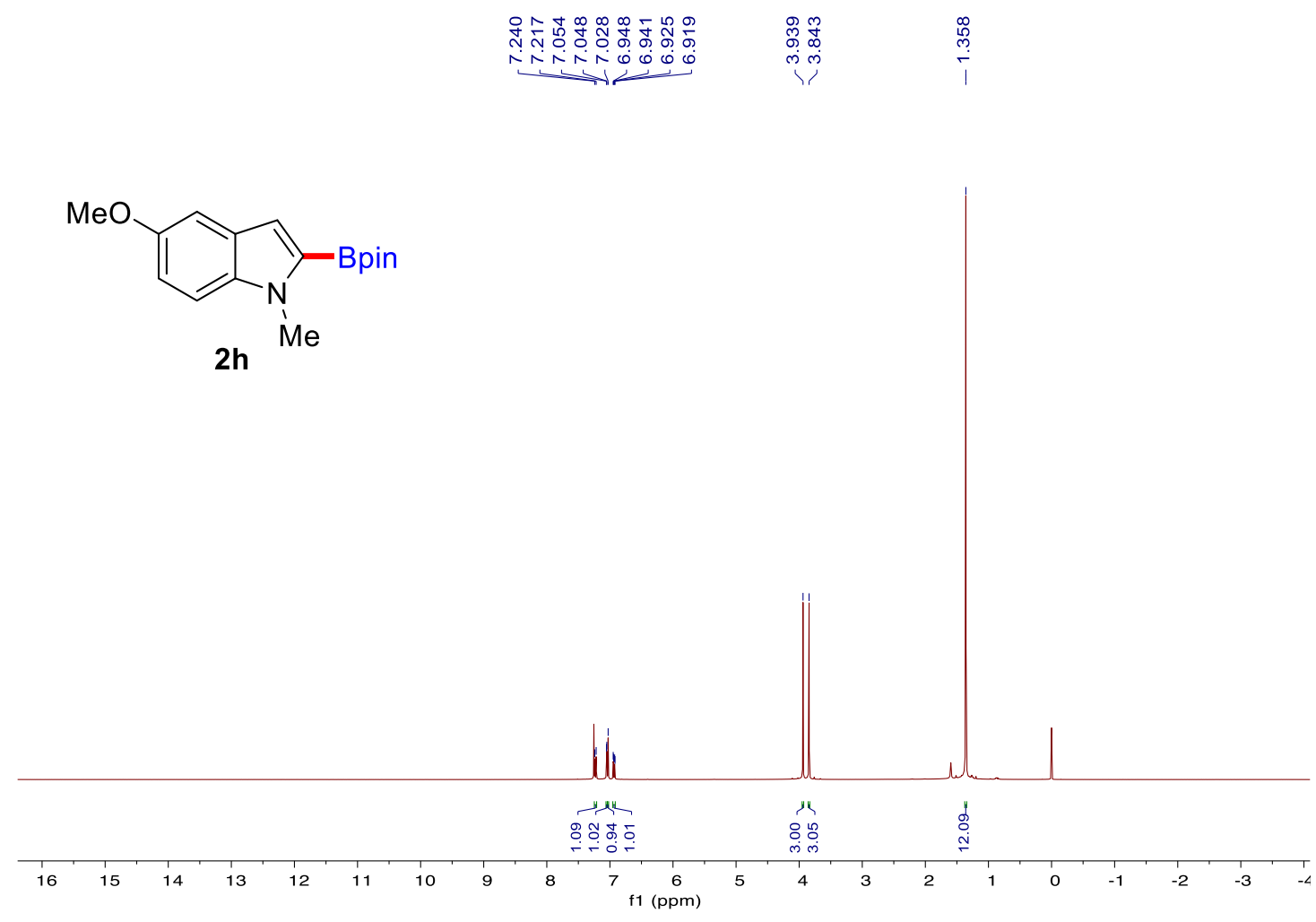

$$
\begin{aligned}
& { }^{13} \mathrm{C} \mathrm{NMR} \mathrm{spectrum} \mathrm{of} \mathbf{2 h} \text { in } \mathrm{CDCl}_{3}(100 \mathrm{MHz})
\end{aligned}
$$

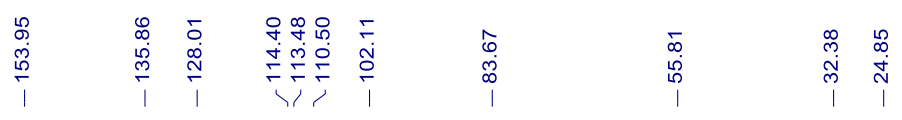<smiles>COc1ccc2c(c1)cc(Cc1ccccc1)n2C</smiles>
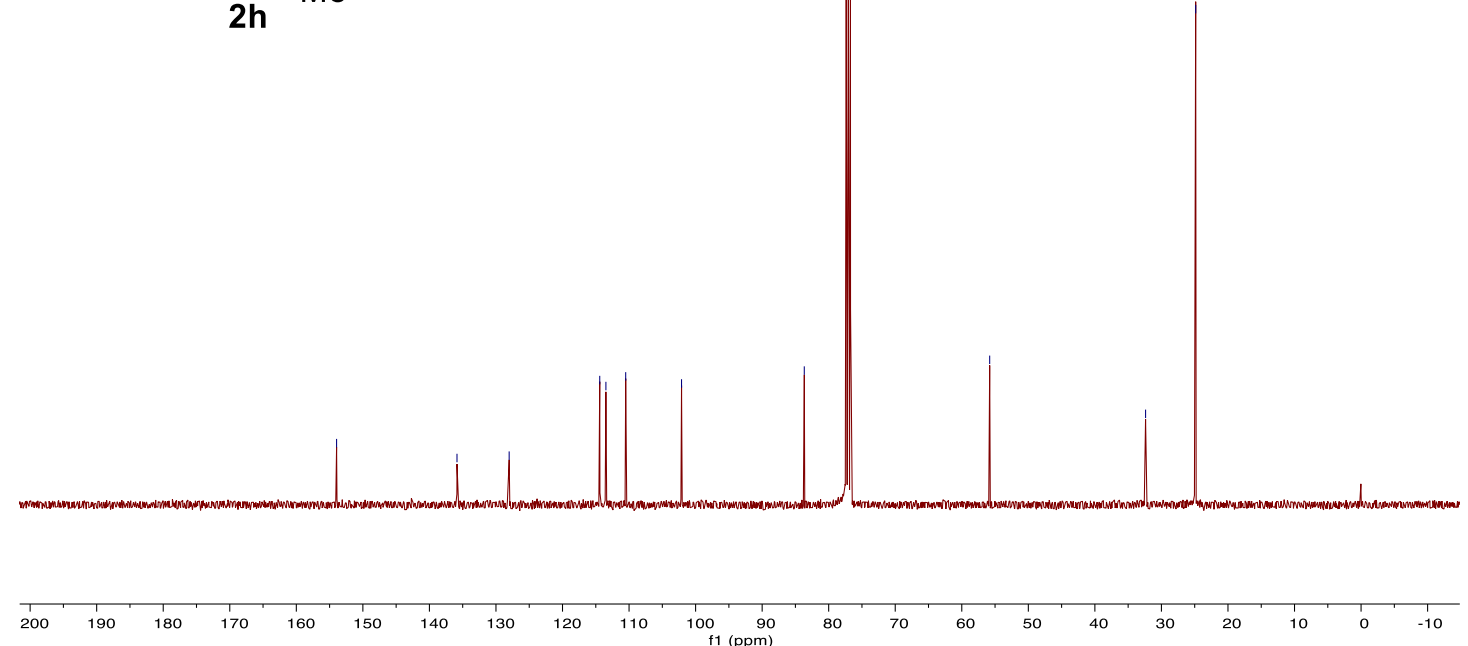
${ }^{1} \mathrm{H}$ NMR spectrum of $2 \mathbf{i}$ in $\mathrm{CDCl}_{3}(400 \mathrm{MHz})$

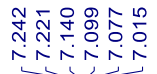

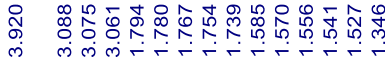

Bpin

2i
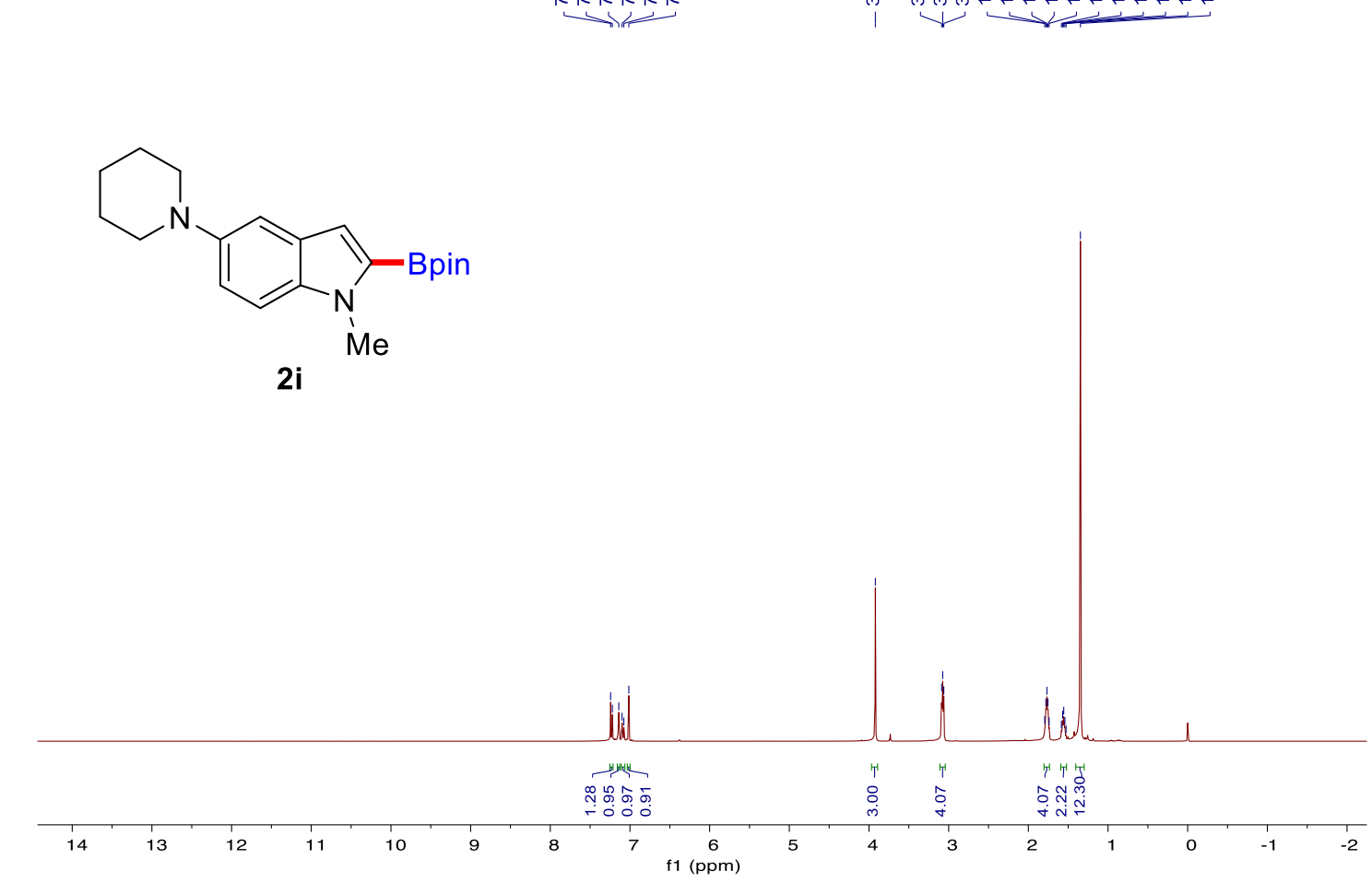

${ }^{13} \mathrm{C}$ NMR spectrum of $2 \mathbf{i}$ in $\mathrm{CDCl}_{3}(100 \mathrm{MHz})$

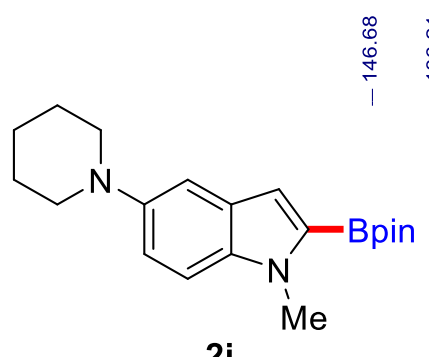

$\mathbf{2 i}$

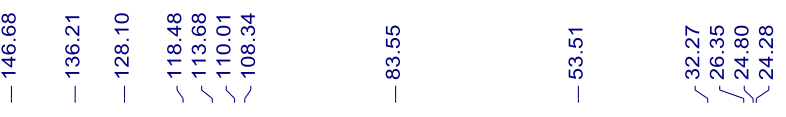

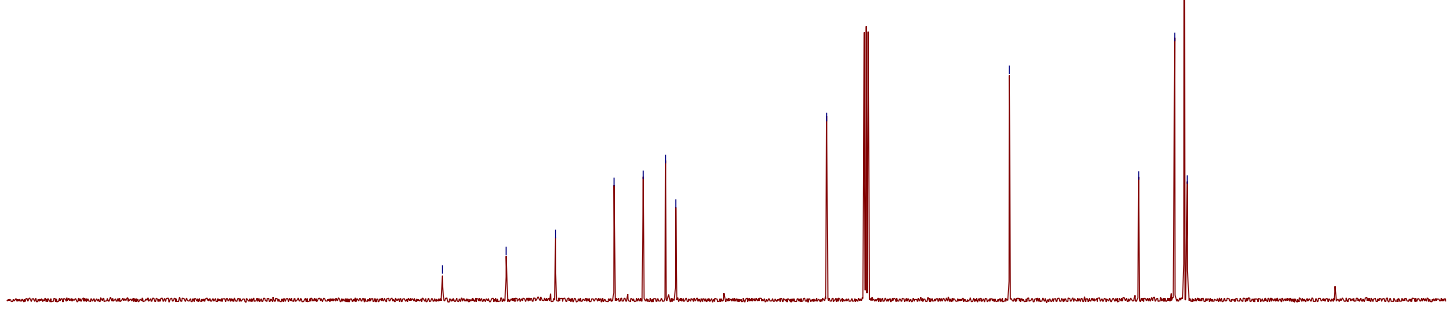

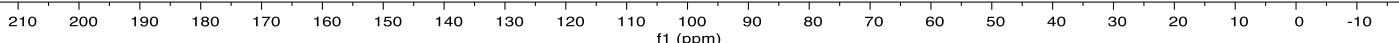


${ }^{1} \mathrm{H}$ NMR spectrum of $\mathbf{2} \mathbf{j}$ in $\mathrm{CDCl}_{3}(400 \mathrm{MHz})$

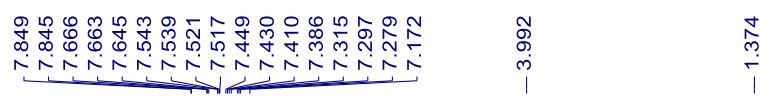

Bpin

2j
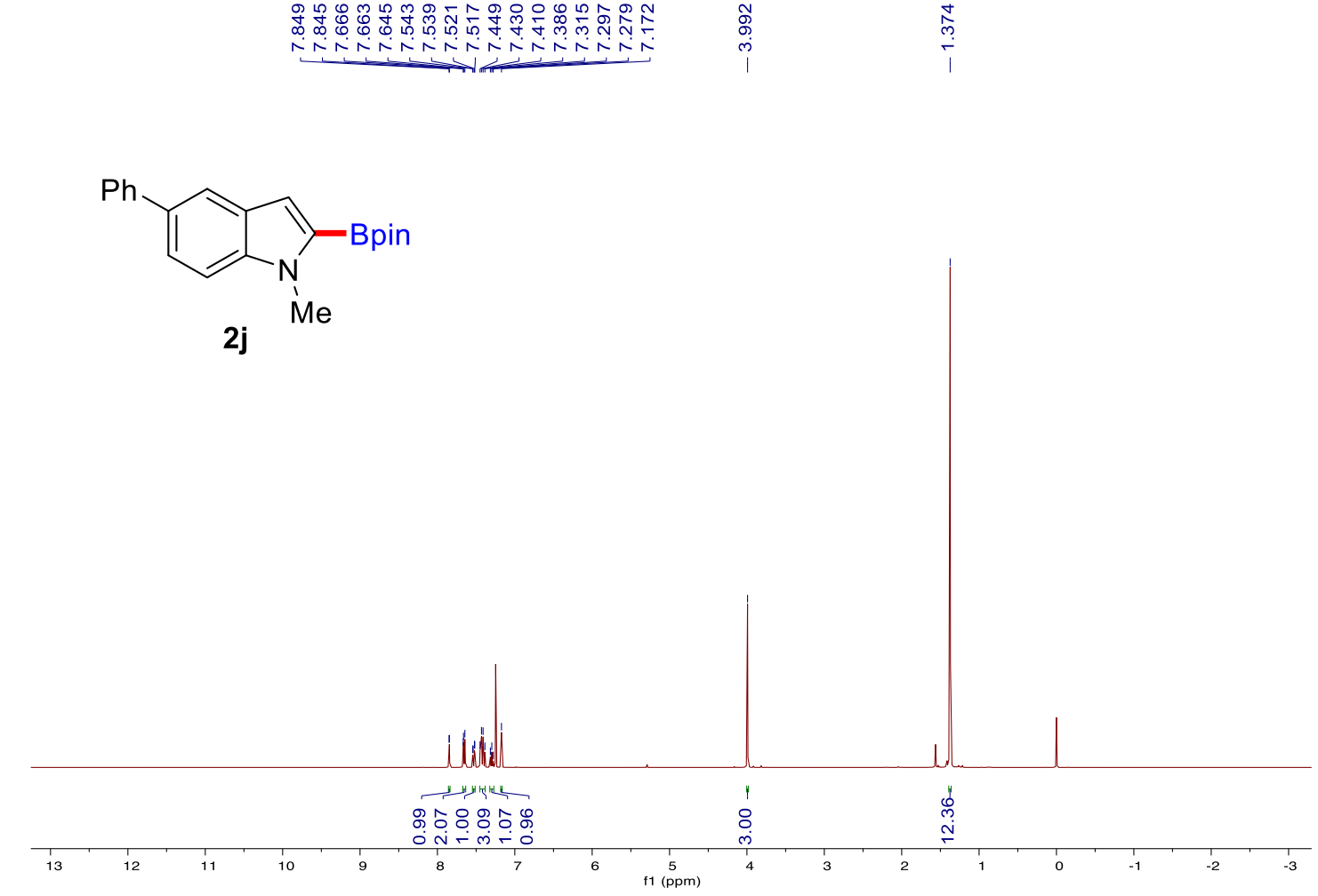

${ }^{13} \mathrm{C} \mathrm{NMR}$ spectrum of $\mathbf{2} \mathbf{j}$ in $\mathrm{CDCl}_{3}(100 \mathrm{MHz})$

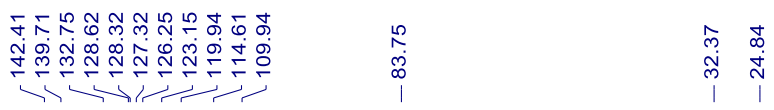

Bpin

2j

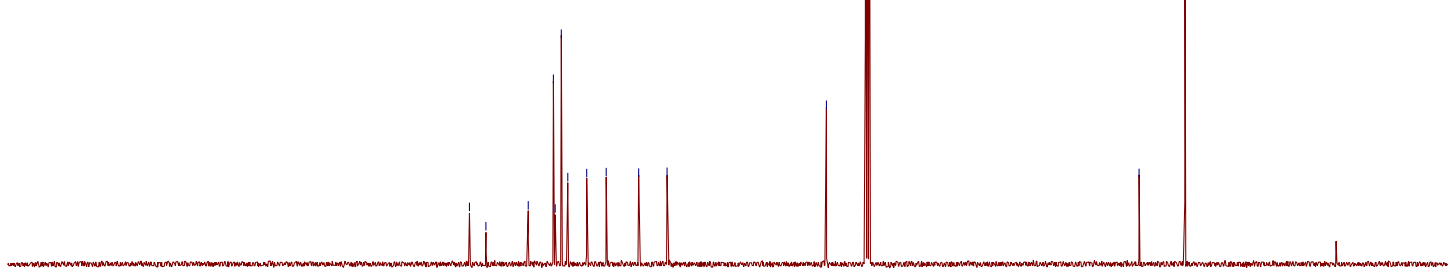

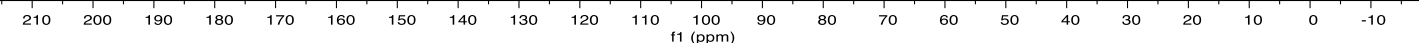


${ }^{1} \mathrm{H}$ NMR spectrum of $\mathbf{2} \mathbf{k}$ in $\mathrm{CDCl}_{3}(400 \mathrm{MHz})$

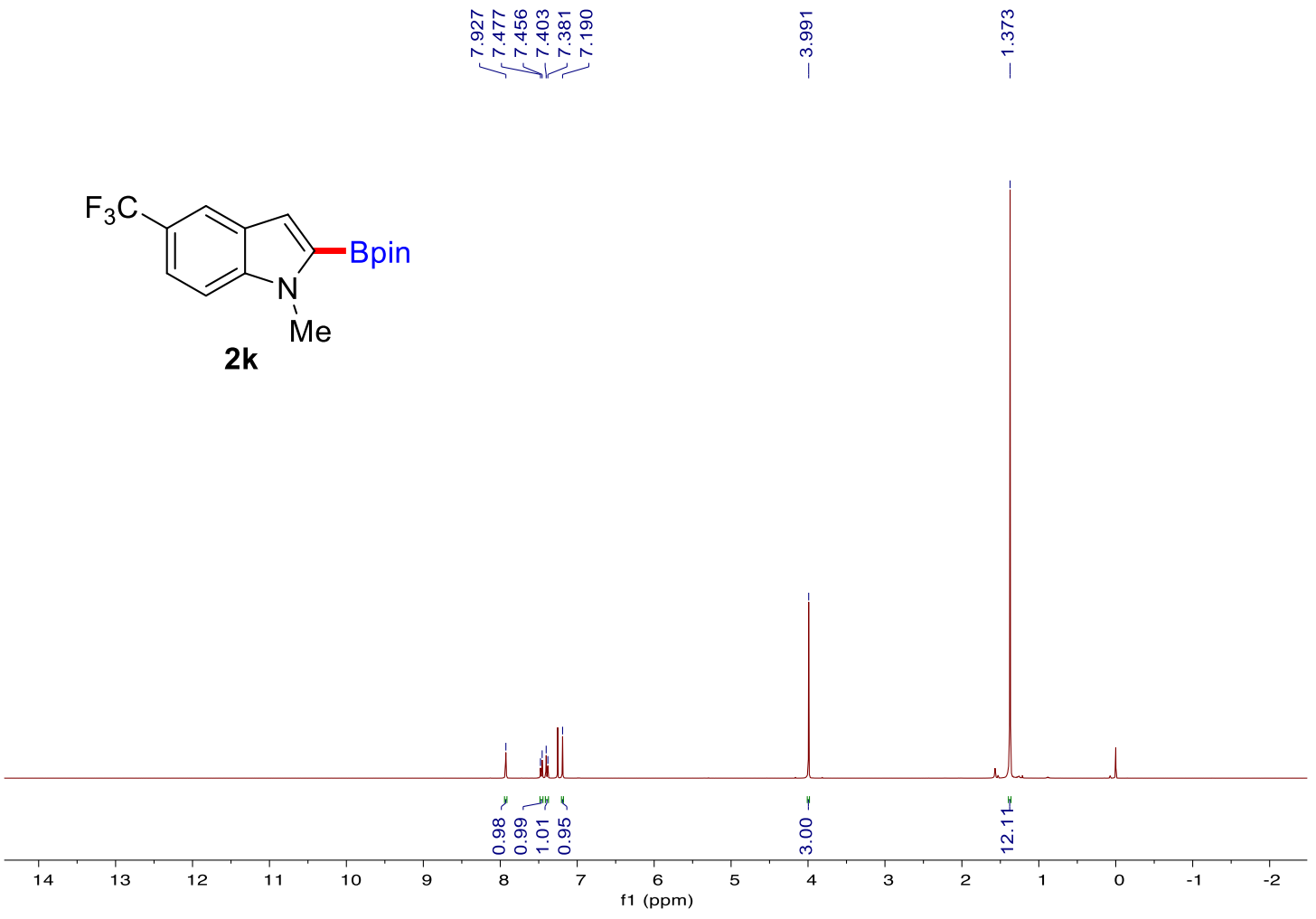

${ }^{13} \mathrm{C} \mathrm{NMR}$ spectrum of $\mathbf{2} \mathbf{k}$ in $\mathrm{CDCl}_{3}(100 \mathrm{MHz})$

0
$m$
0

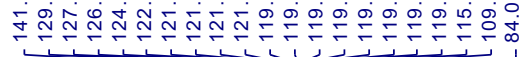

$\begin{array}{cc}\infty & + \\ \stackrel{\infty}{+} & \infty \\ & \stackrel{d}{+} \\ 1 & \mid\end{array}$

2k<smiles>Cn1c(CCc2ccccc2)cc2cc(C(F)(F)F)ccc21</smiles>

$\begin{array}{lllllllllllllllllllllllll}1 & 210 & 200 & 190 & 180 & 170 & 160 & 150 & 140 & 130 & 120 & 110 & 100 & 90 & 80 & 70 & 60 & 50 & 40 & 30 & 20 & 10 & 0 & -10\end{array}$ 
${ }^{1} \mathrm{H}$ NMR spectrum of $\mathbf{2 l}$ in $\mathrm{CDCl}_{3}(400 \mathrm{MHz})$ |<smiles>Cn1c(Cc2ccccc2)cc2cc(F)ccc21</smiles>

2I $\mathrm{Me}$

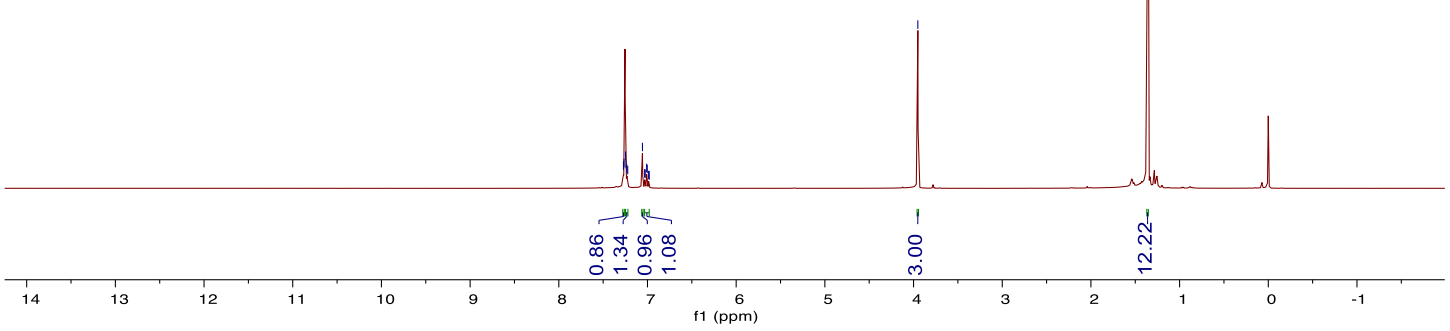

${ }^{13} \mathrm{C}$ NMR spectrum of $2 \mathrm{l}$ in $\mathrm{CDCl}_{3}(100 \mathrm{MHz})$

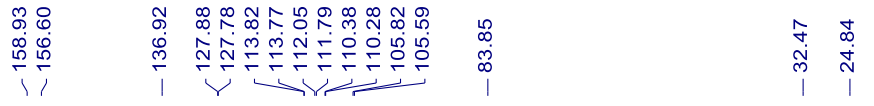<smiles>Cn1c(Cc2ccccc2)cc2cc(F)ccc21</smiles>

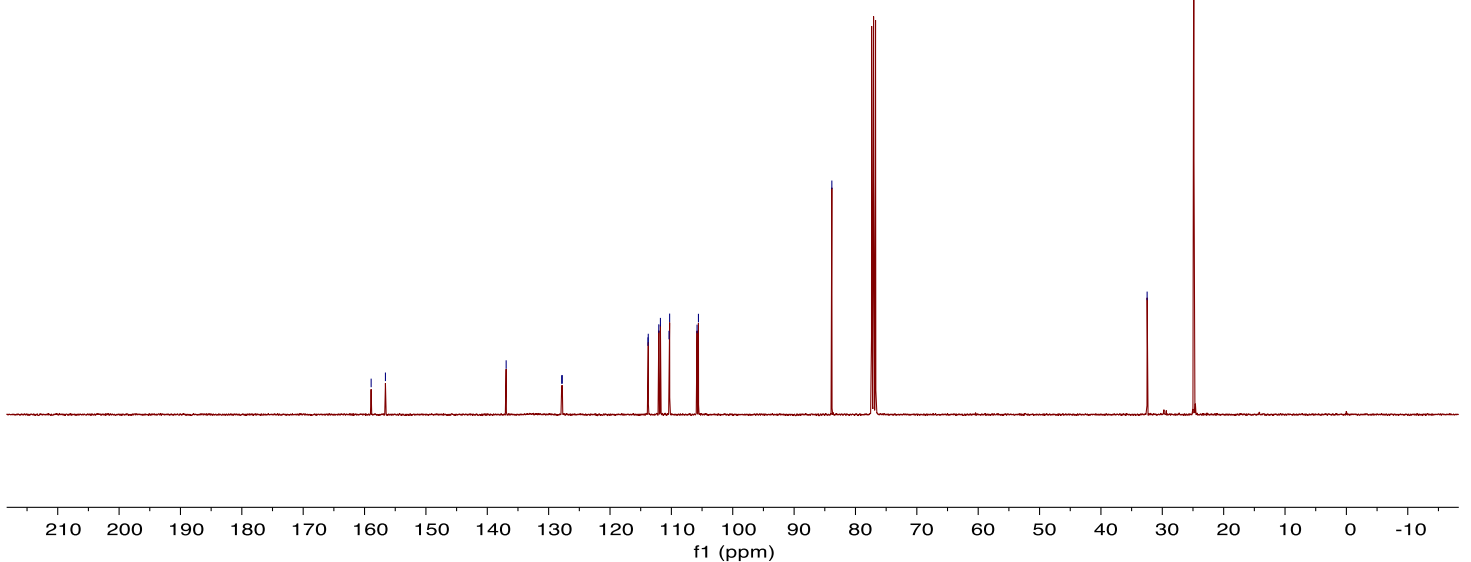


${ }^{1} \mathrm{H}$ NMR spectrum of $\mathbf{2} \mathbf{m}$ in $\mathrm{CDCl}_{3}(400 \mathrm{MHz})$
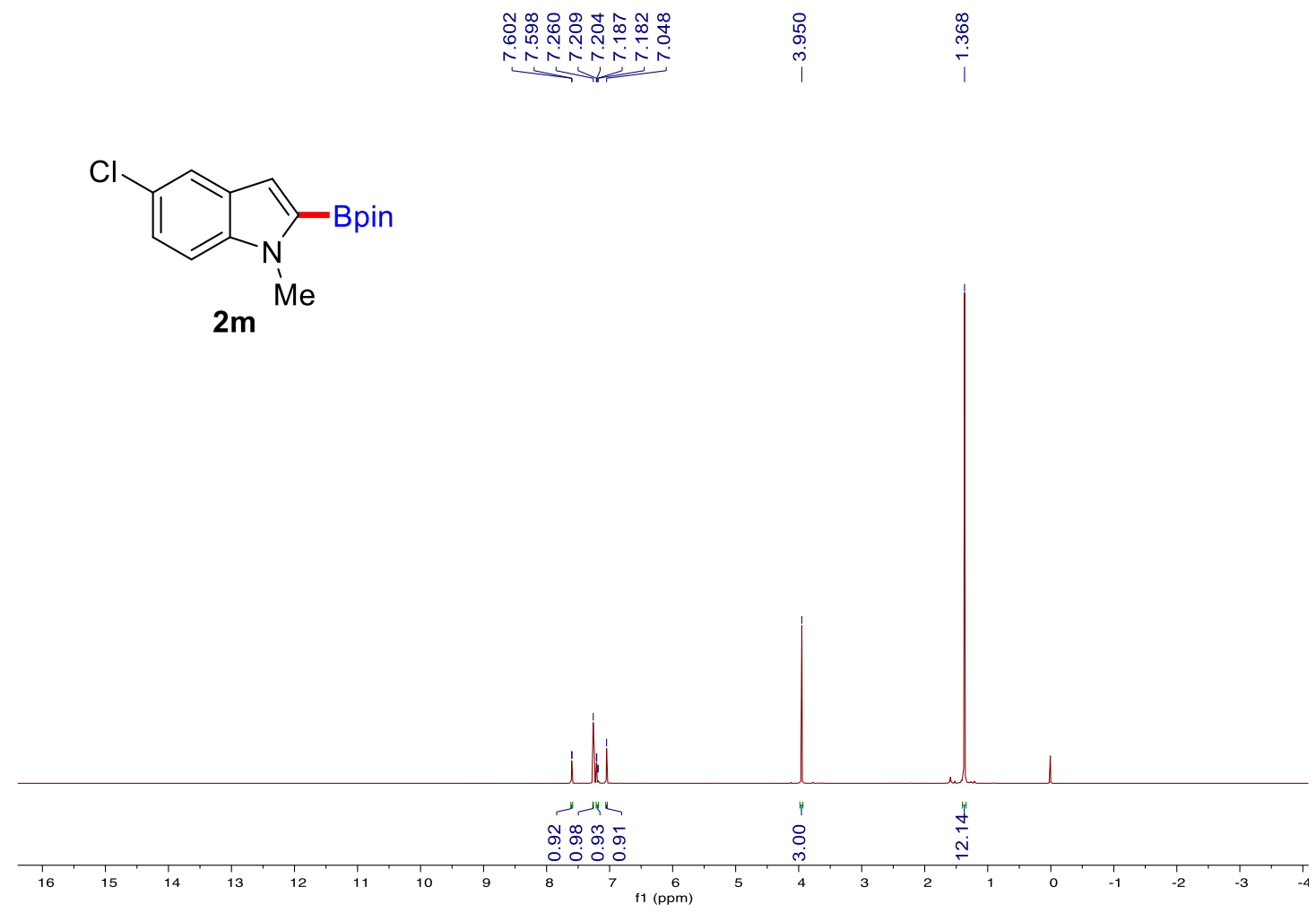

${ }^{13} \mathrm{C}$ NMR spectrum of $\mathbf{2} \mathbf{m}$ in $\mathrm{CDCl}_{3}(100 \mathrm{MHz})$

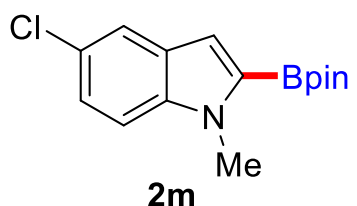

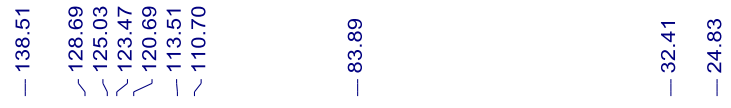

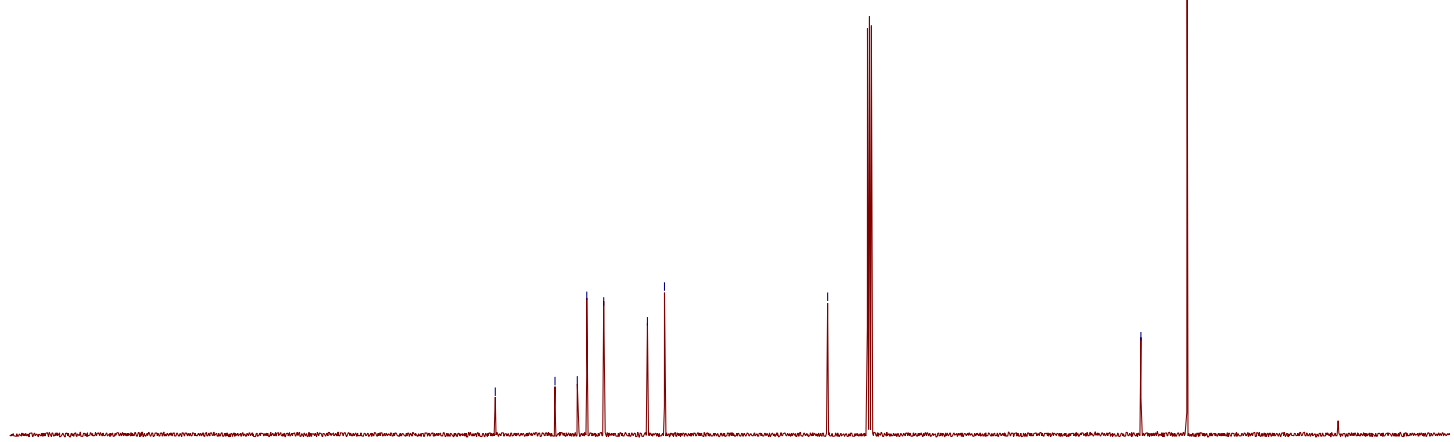

$\begin{array}{lllllllllllllllllllllll}210 & 200 & 190 & 180 & 170 & 160 & 150 & 140 & 130 & 120 & 110 & \begin{array}{l}100 \\ 100\end{array} & 90 & 80 & 70 & 60 & 50 & 40 & 30 & 20 & 10 & 0 & -10\end{array}$ 
${ }^{1} \mathrm{H}$ NMR spectrum of $\mathbf{2 n}$ in $\mathrm{CDCl}_{3}(400 \mathrm{MHz})$

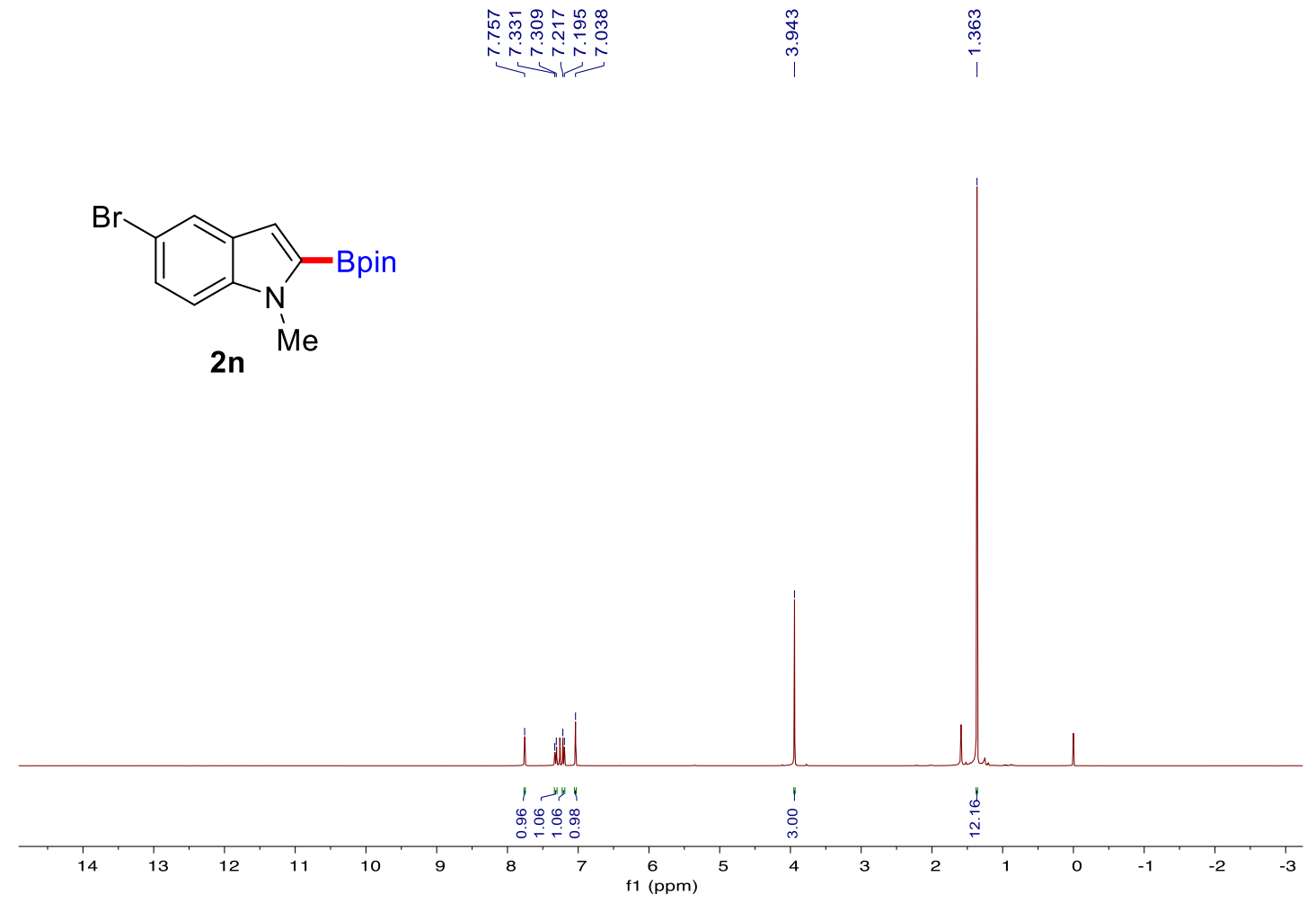

${ }^{13} \mathrm{C}$ NMR spectrum of $\mathbf{2 n}$ in $\mathrm{CDCl}_{3}(100 \mathrm{MHz})$

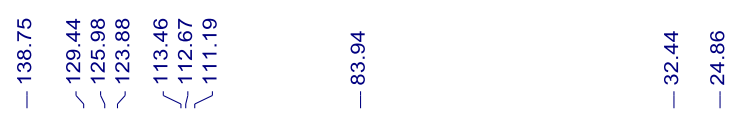<smiles>Cn1c(Cc2ccccc2)cc2cc(Br)ccc21</smiles>

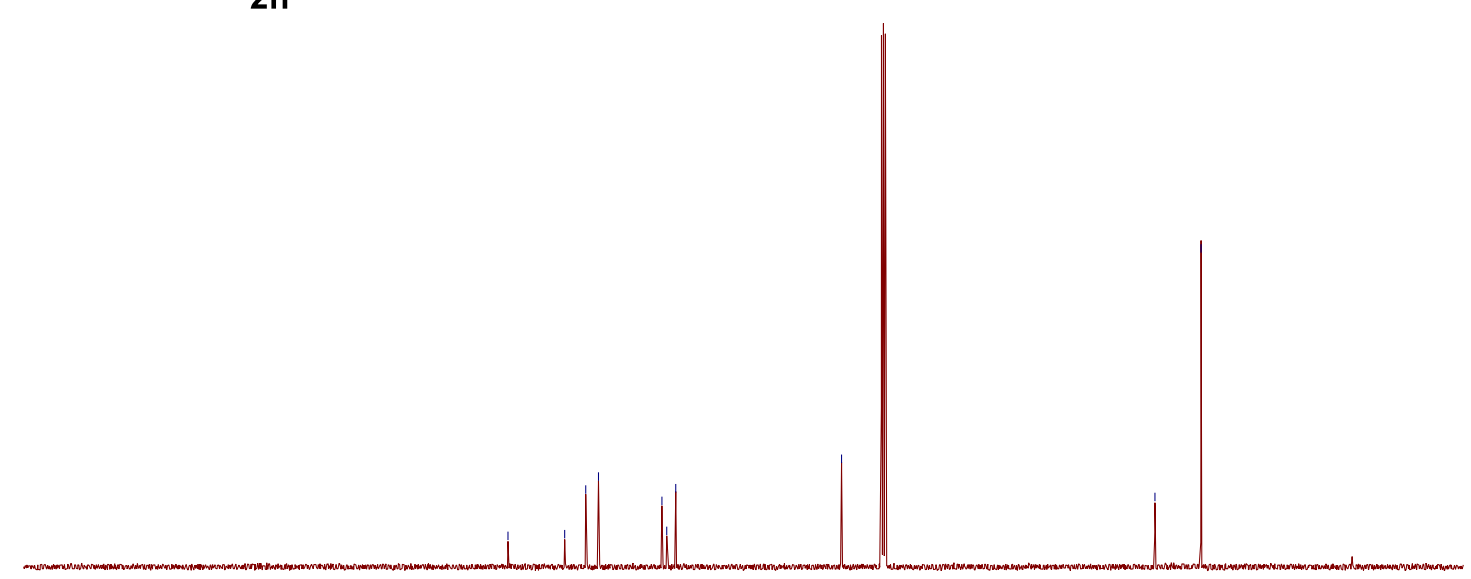


${ }^{1} \mathrm{H}$ NMR spectrum of $2 \mathrm{o}$ in $\mathrm{CDCl}_{3}(400 \mathrm{MHz})$
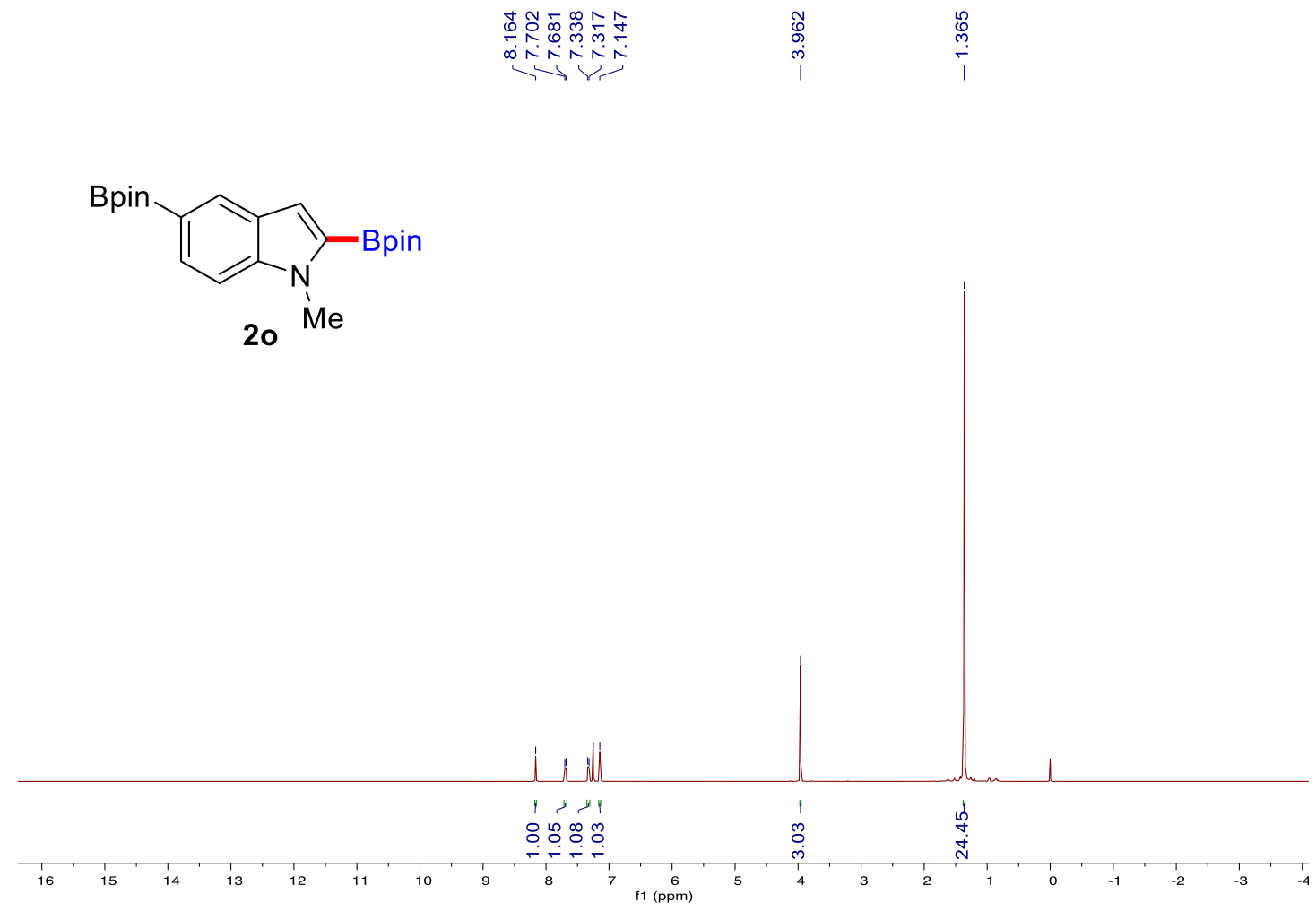

${ }^{13} \mathrm{C}$ NMR spectrum of 20 in $\mathrm{CDCl}_{3}(100 \mathrm{MHz})$

\begin{tabular}{|c|c|}
\hline 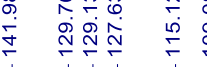 & 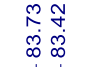 \\
\hline
\end{tabular}

Bpin<smiles>c1ccc(Cc2cc3ccccc3[nH]2)cc1</smiles>

$20 \mathrm{Me}$

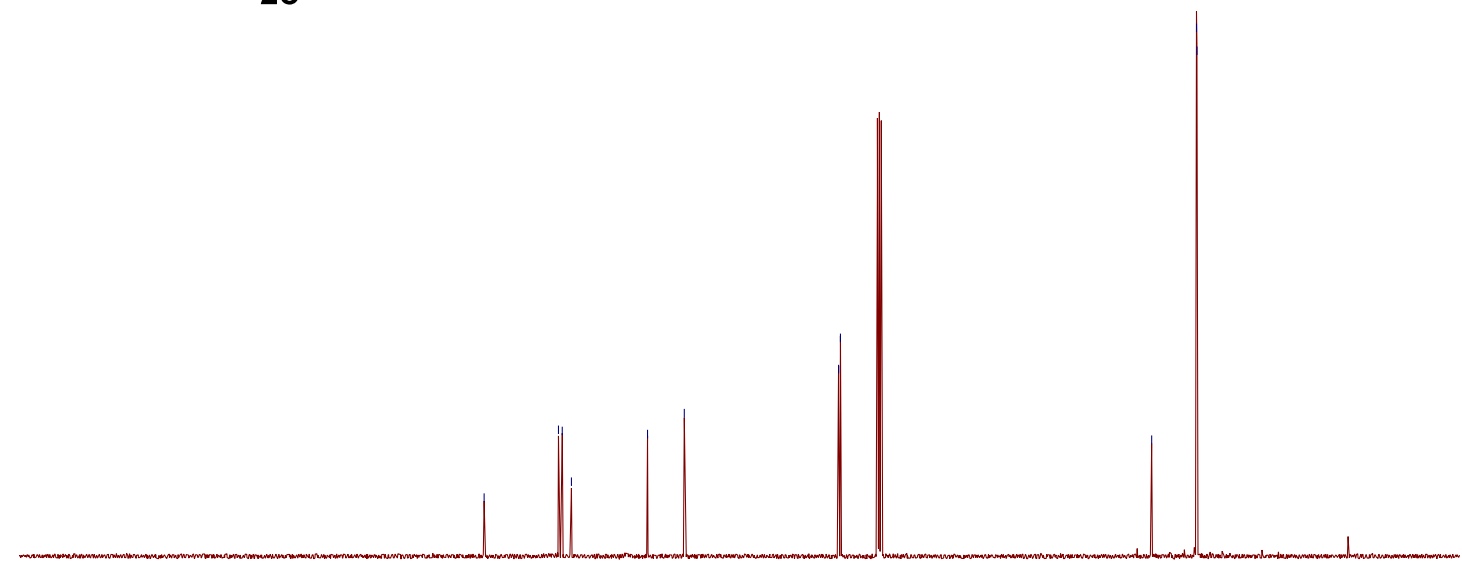

$\begin{array}{lllllllllllllllllllllll}210 & 200 & 190 & 180 & 170 & 160 & 150 & 140 & 130 & 120 & 110 & 100 & 90 & 80 & 70 & 60 & 50 & 40 & 30 & 20 & 10 & 0 & -10\end{array}$ 
${ }^{1} \mathrm{H}$ NMR spectrum of $\mathbf{2 p}$ in $\mathrm{CDCl}_{3}(400 \mathrm{MHz})$
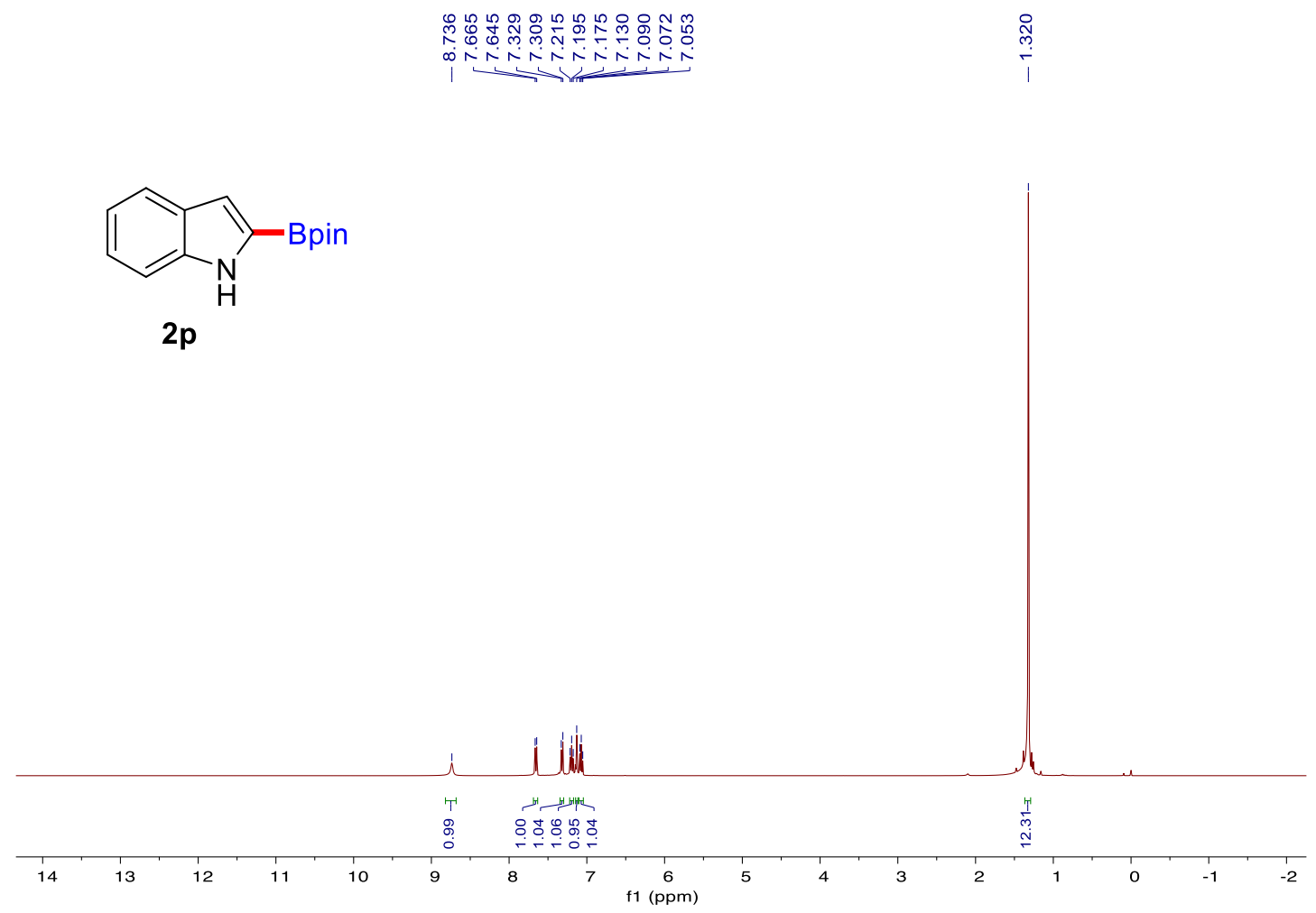

${ }^{13} \mathrm{C}$ NMR spectrum of $\mathbf{2 p}$ in $\mathrm{CDCl}_{3}(100 \mathrm{MHz})$

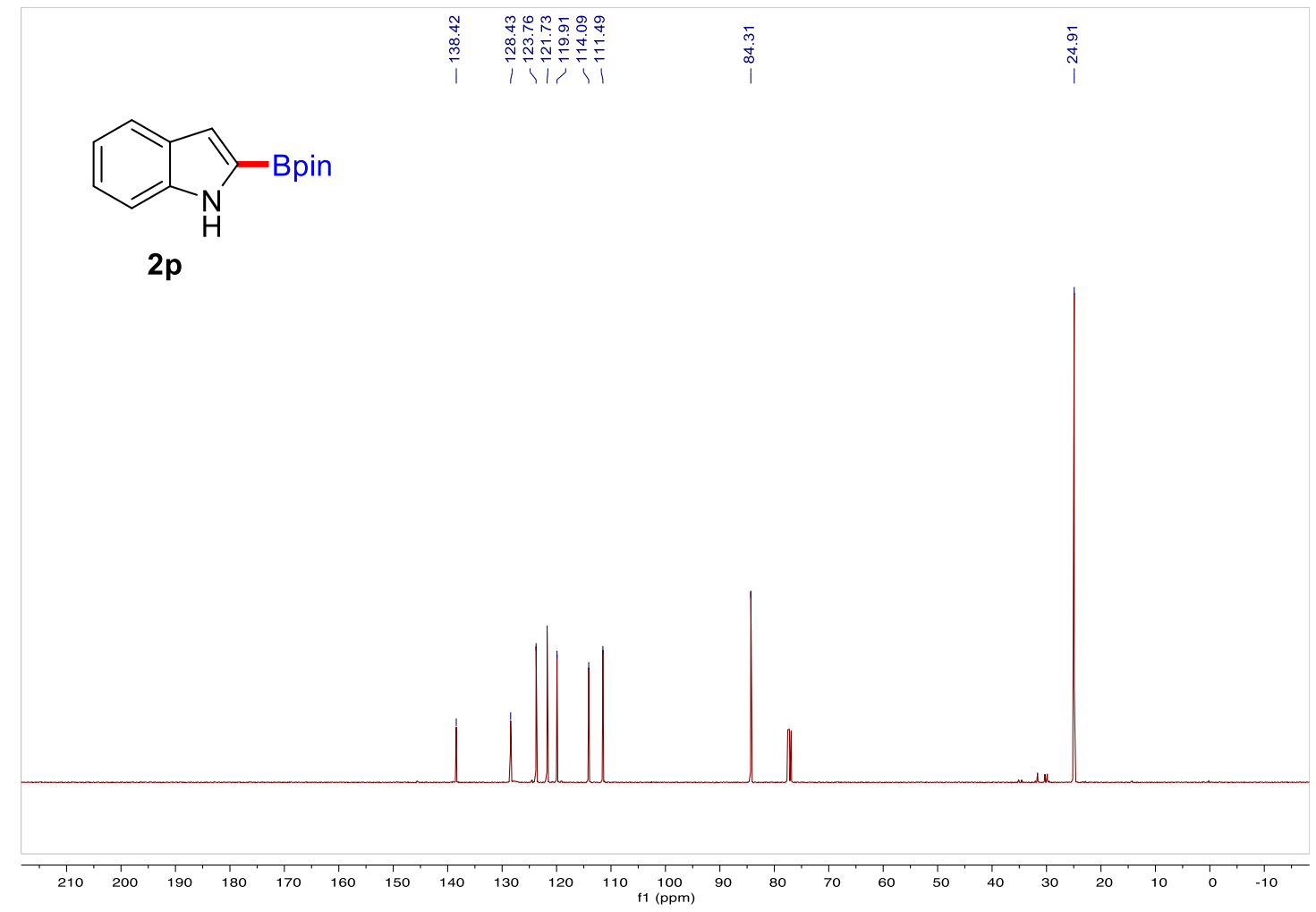


${ }^{1} \mathrm{H}$ NMR spectrum of $\mathbf{2 q}$ in $\mathrm{CDCl}_{3}(400 \mathrm{MHz})$
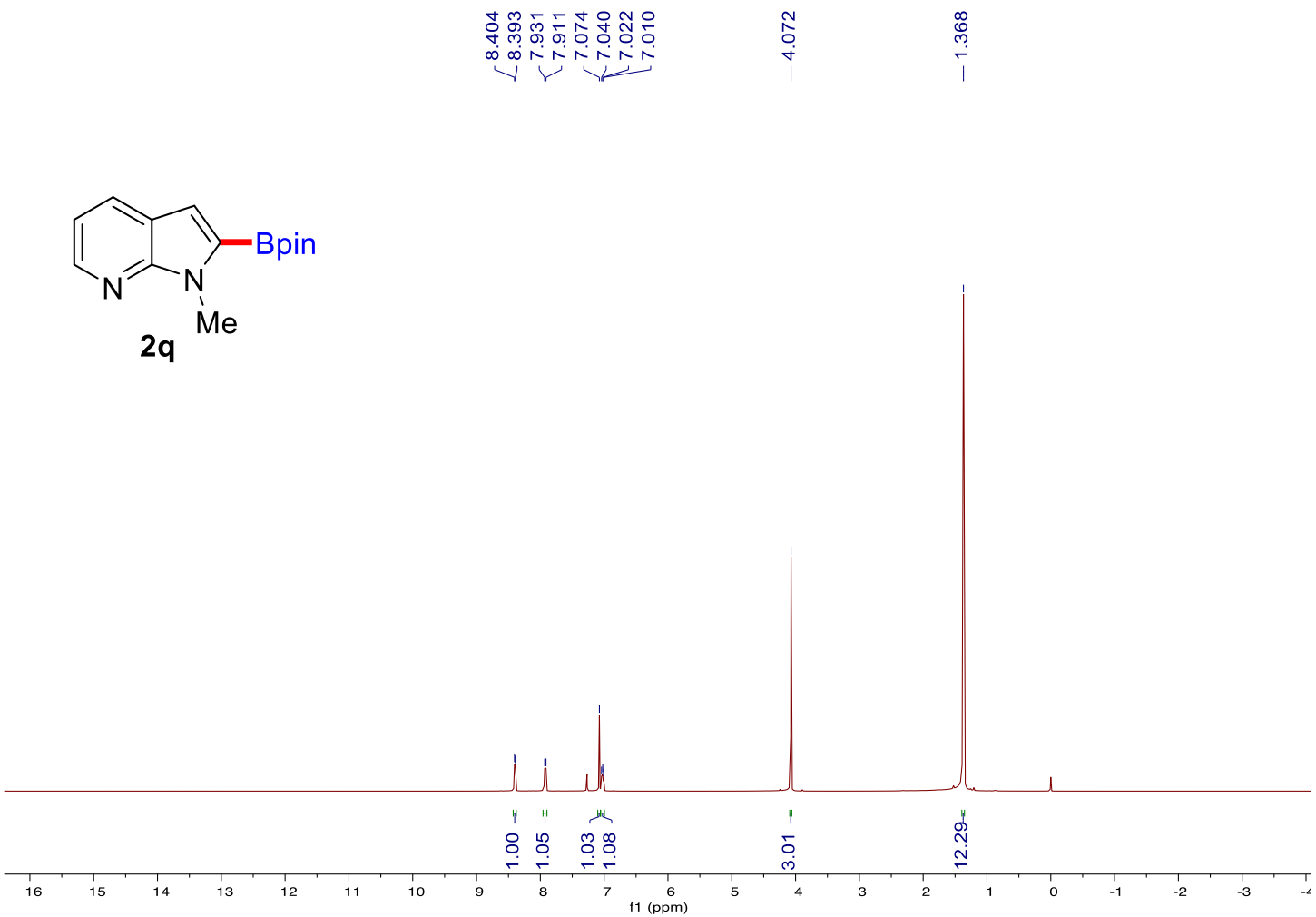

${ }^{13} \mathrm{C}$ NMR spectrum of $\mathbf{2 q}$ in $\mathrm{CDCl}_{3}(100 \mathrm{MHz})$

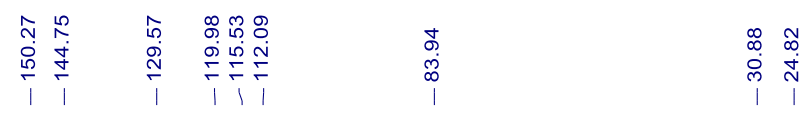<smiles>Cn1c(Cc2ccccc2)cc2cccnc21</smiles>

$2 q$

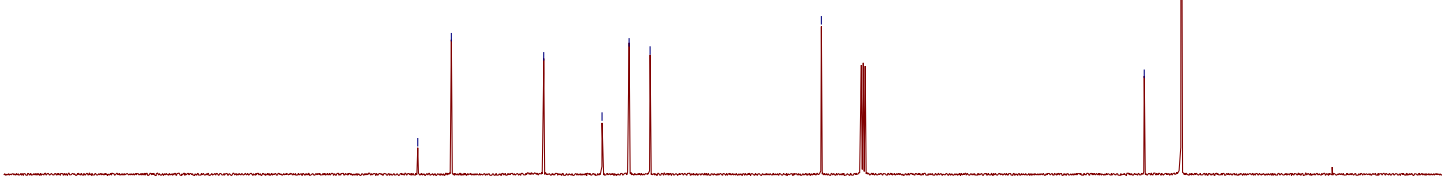

$\begin{array}{llllllllllllllllllllllllllllllll} & 210 & 200 & 190 & 180 & 170 & 160 & 150 & 140 & 130 & 120 & 110 & 100 & 90 & 80 & 70 & 60 & 50 & 40 & 30 & 20 & 10 & 0 & -10\end{array}$ 
${ }^{1} \mathrm{H}$ NMR spectrum of $\mathbf{2} \mathbf{r}$ in $\mathrm{CDCl}_{3}(400 \mathrm{MHz})$

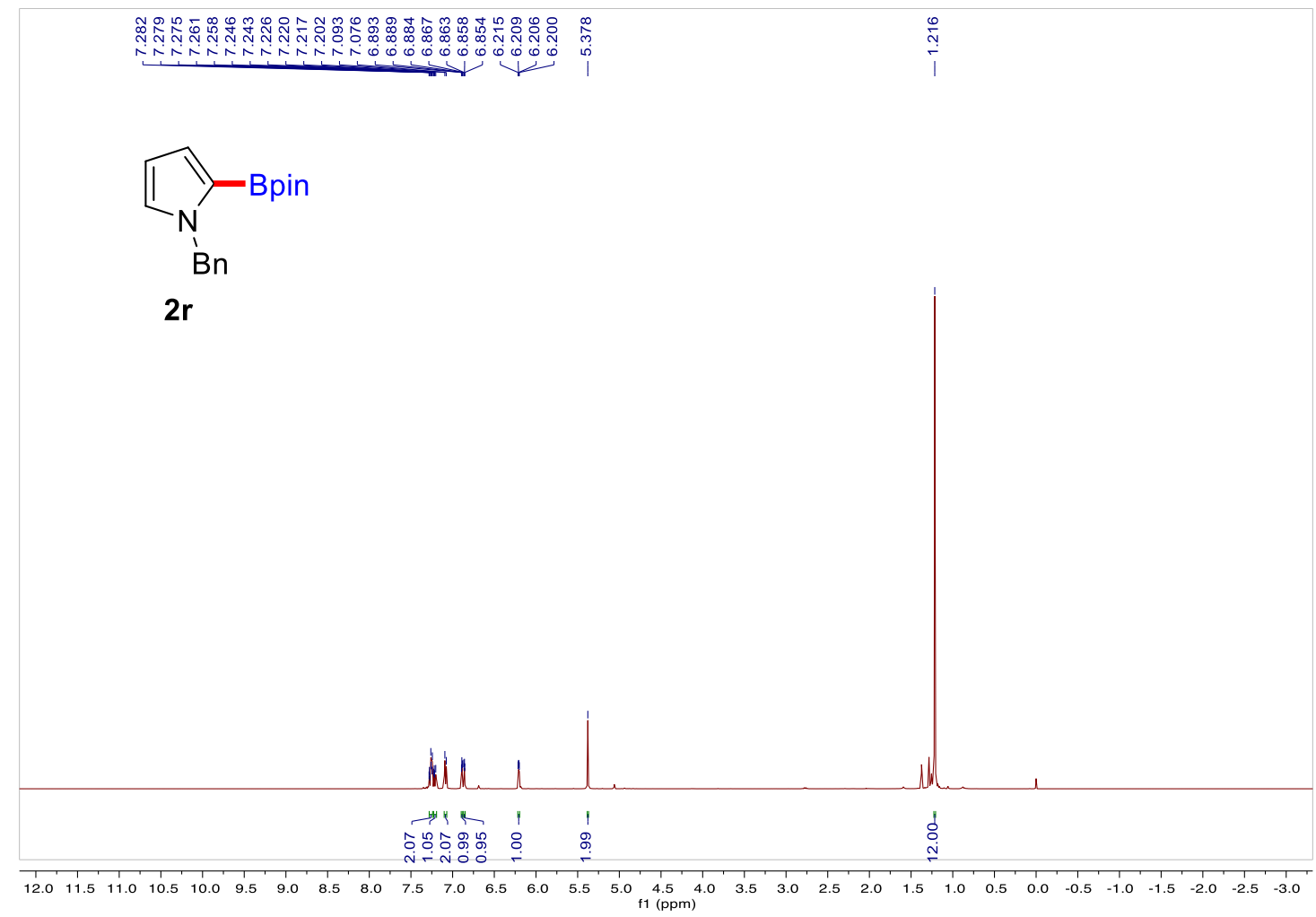

${ }^{13} \mathrm{C}$ NMR spectrum of $2 \mathbf{r}$ in $\mathrm{CDCl}_{3}(100 \mathrm{MHz})$

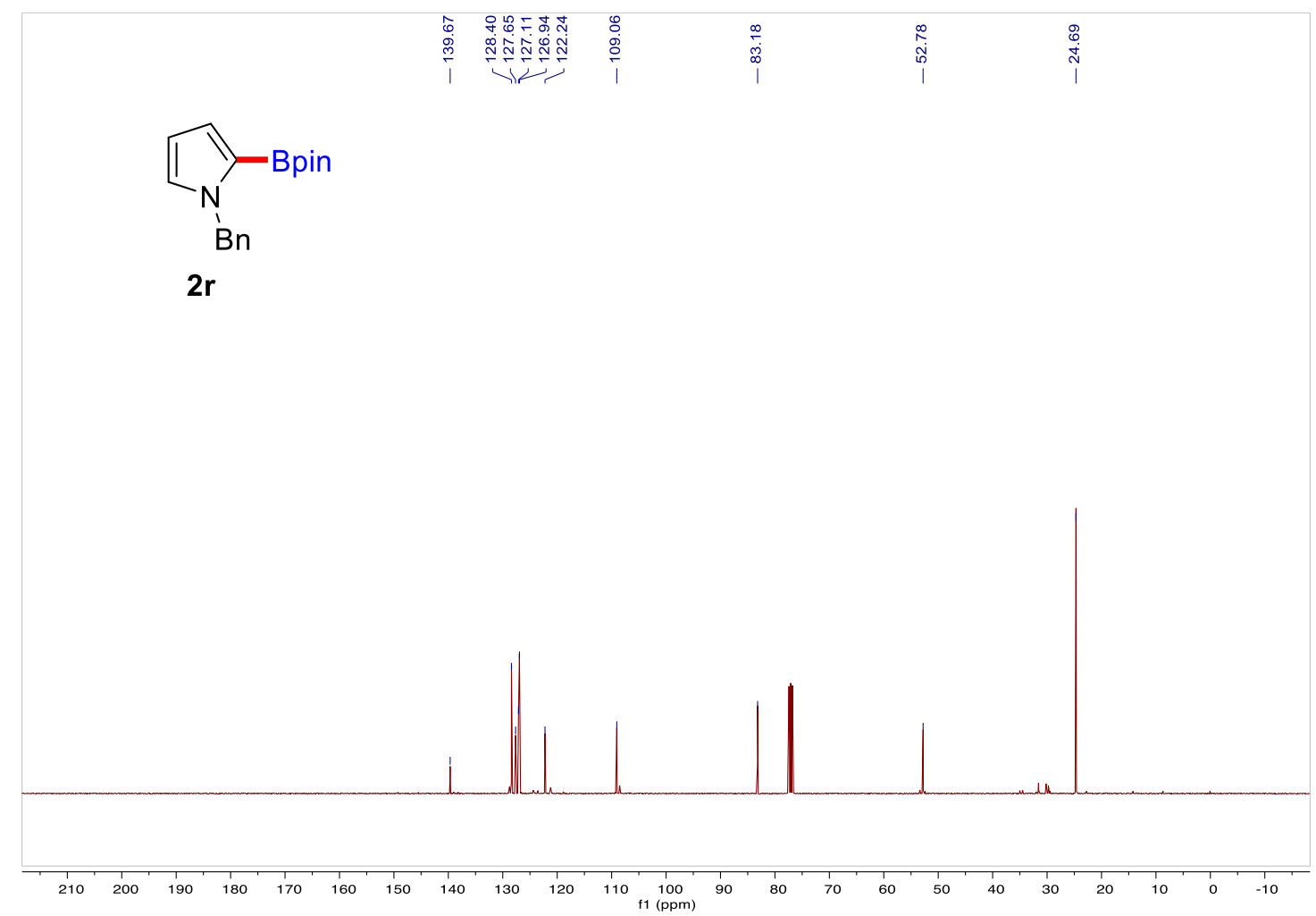


${ }^{1} \mathrm{H}$ NMR spectrum of crude $2 \mathrm{~s}$ in $\mathrm{CDCl}_{3}(400 \mathrm{MHz})$

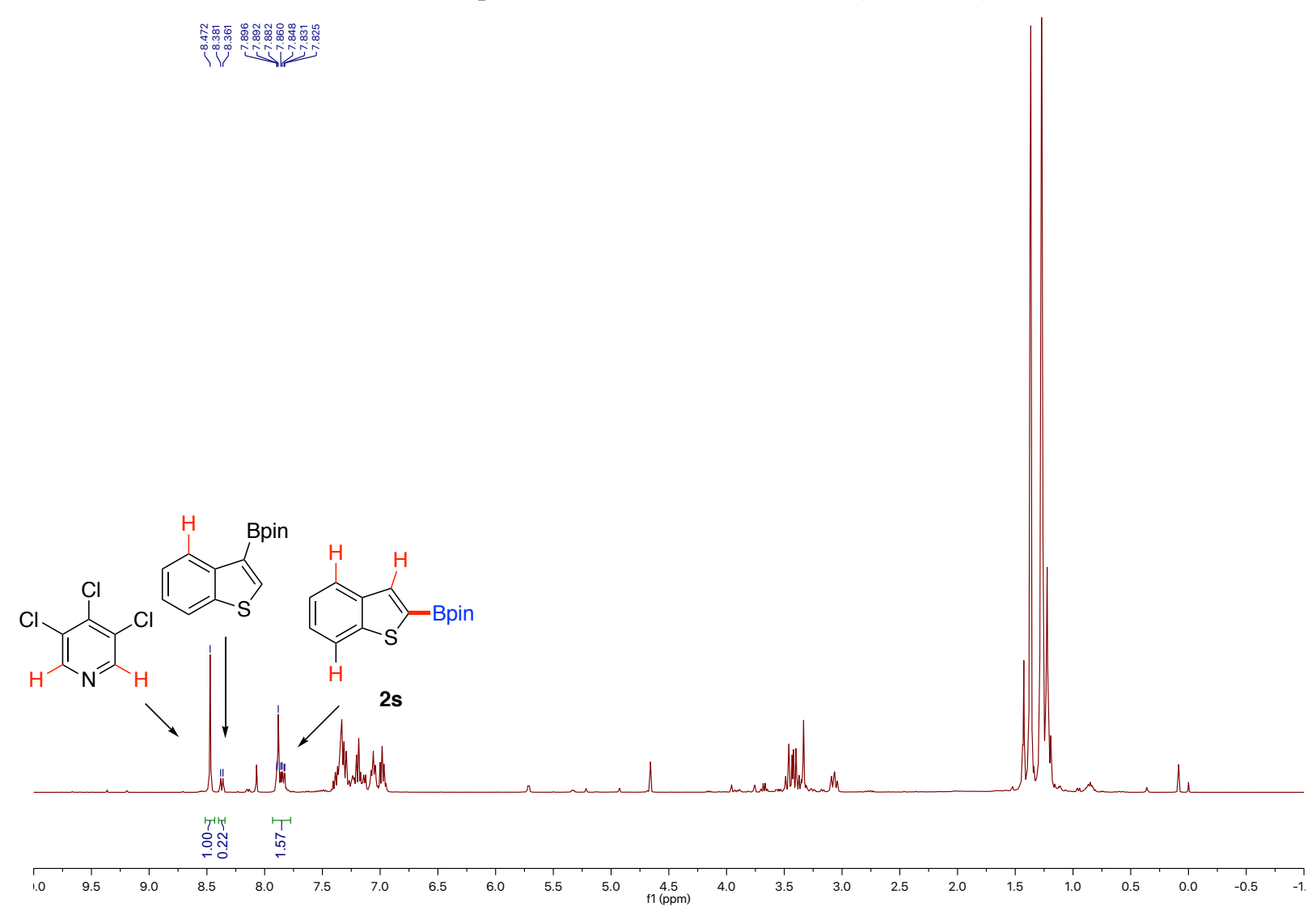

${ }^{1} \mathrm{H}$ NMR spectrum of crude $2 \mathrm{t}$ in $\mathrm{CDCl}_{3}(400 \mathrm{MHz})$

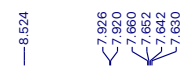

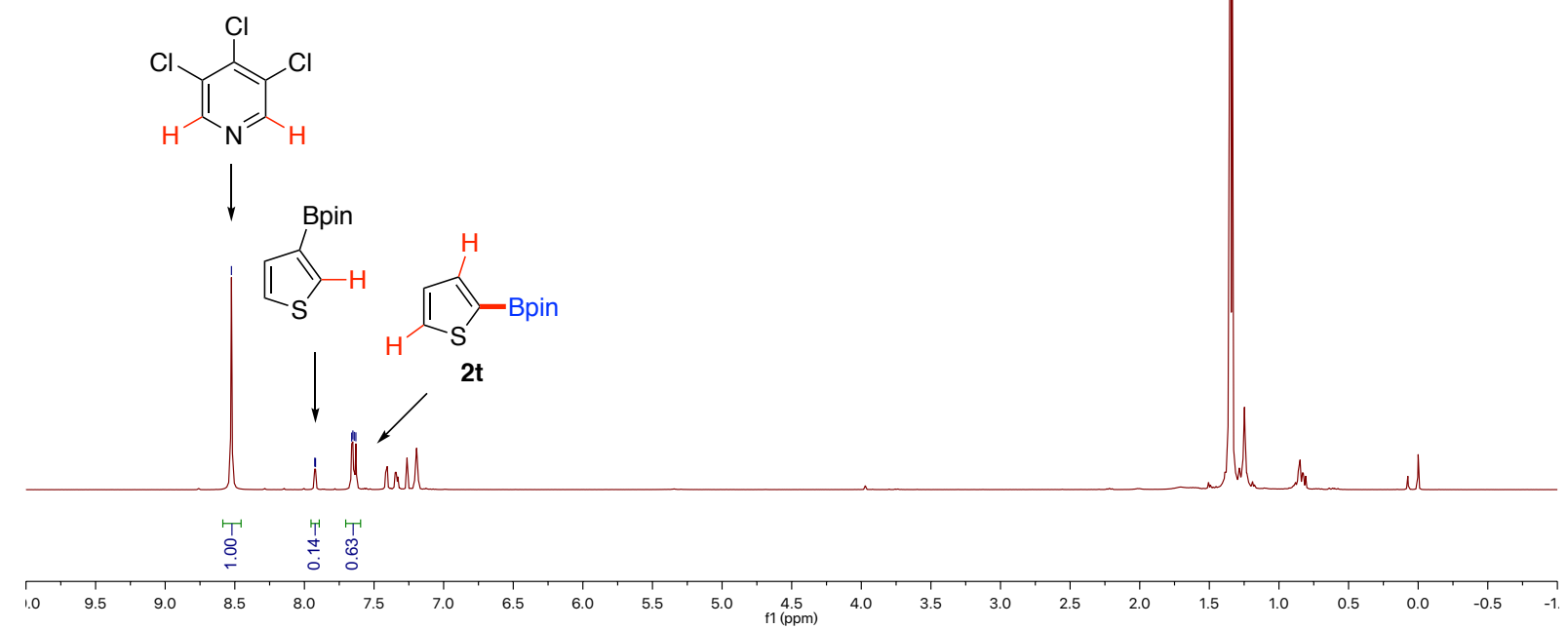


${ }^{1} \mathrm{H}$ NMR spectrum of 5 in $\mathrm{CDCl}_{3}(400 \mathrm{MHz})$

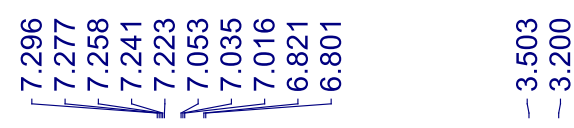

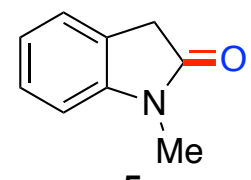

5

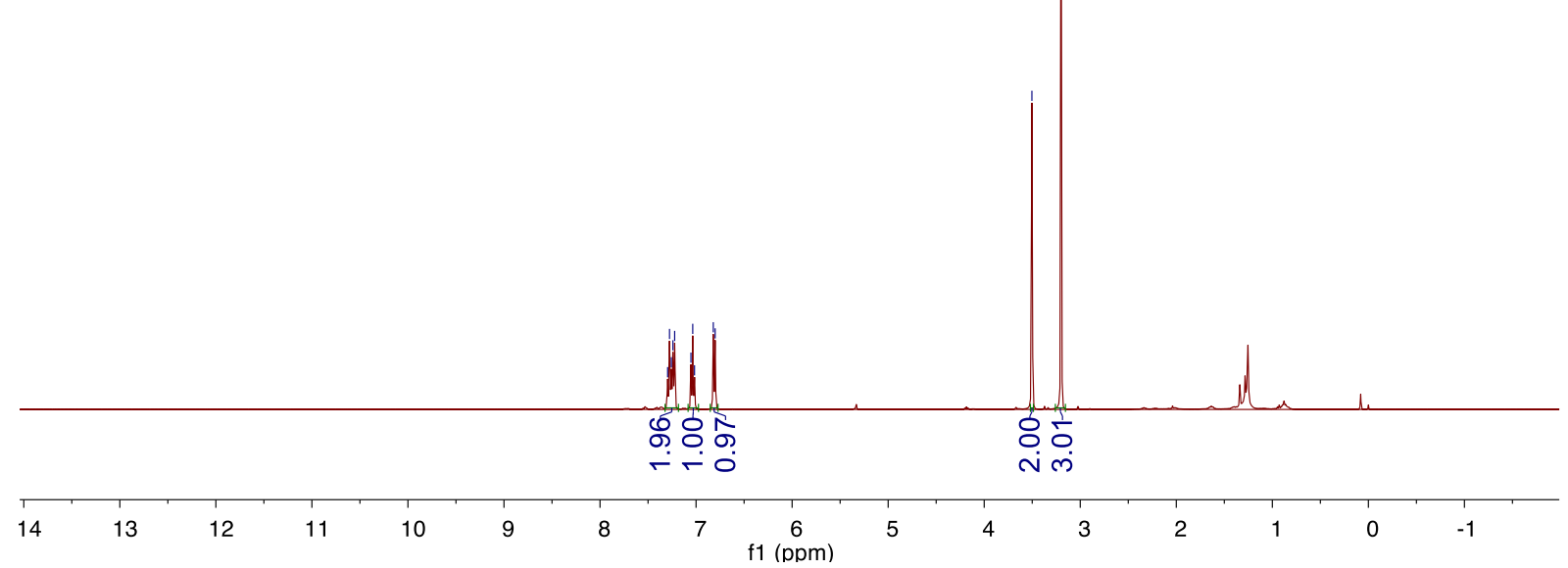

${ }^{13} \mathrm{C}$ NMR spectrum of 5 in $\mathrm{CDCl}_{3}(100 \mathrm{MHz})$

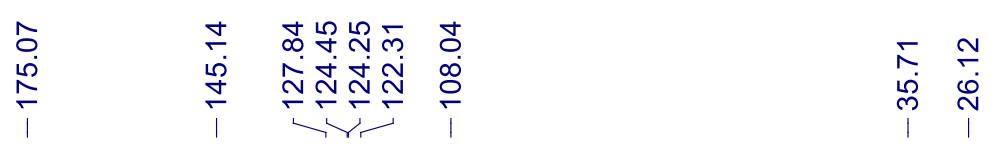<smiles>CN1C(=O)Cc2ccccc21</smiles>

5

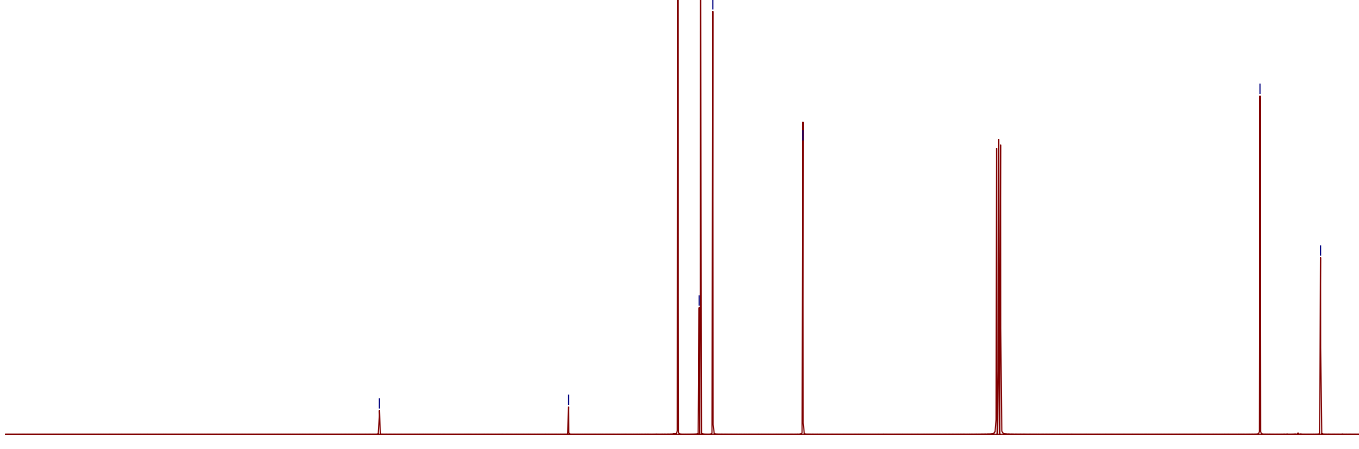

$\begin{array}{lllllllllllllllllllllllllllllllllll}230 & 220 & 210 & 200 & 190 & 180 & 170 & 160 & 150 & 140 & 130 & 120 & 110 & 100 & 90 & 80 & 70 & 60 & 50 & 40 & 30 & 20 & 10 & 0 & -10\end{array}$ 
${ }^{1} \mathrm{H}$ NMR spectrum of 6 in $\mathrm{CDCl}_{3}(400 \mathrm{MHz})$

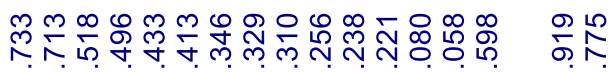

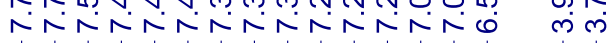

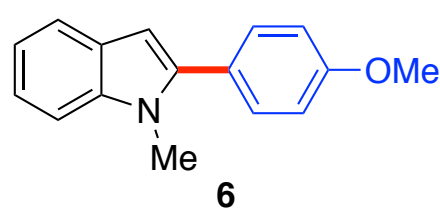

6

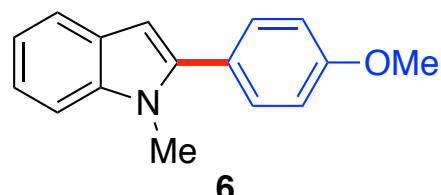

${ }^{13} \mathrm{C}$ NMR spectrum of 6 in $\mathrm{CDCl}_{3}(100 \mathrm{MHz})$

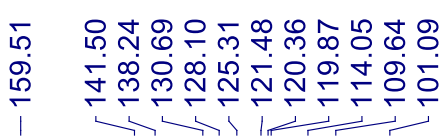

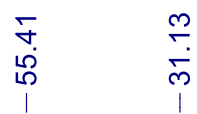

\begin{tabular}{llllllllllllllllll}
\hline 4 & 13 & 12 & 11 & 10 & 9 & 8 & 7 & 6 & 6 & 5 & 4 & 3 & 2 & 1 & 0 & -1 & -1
\end{tabular}

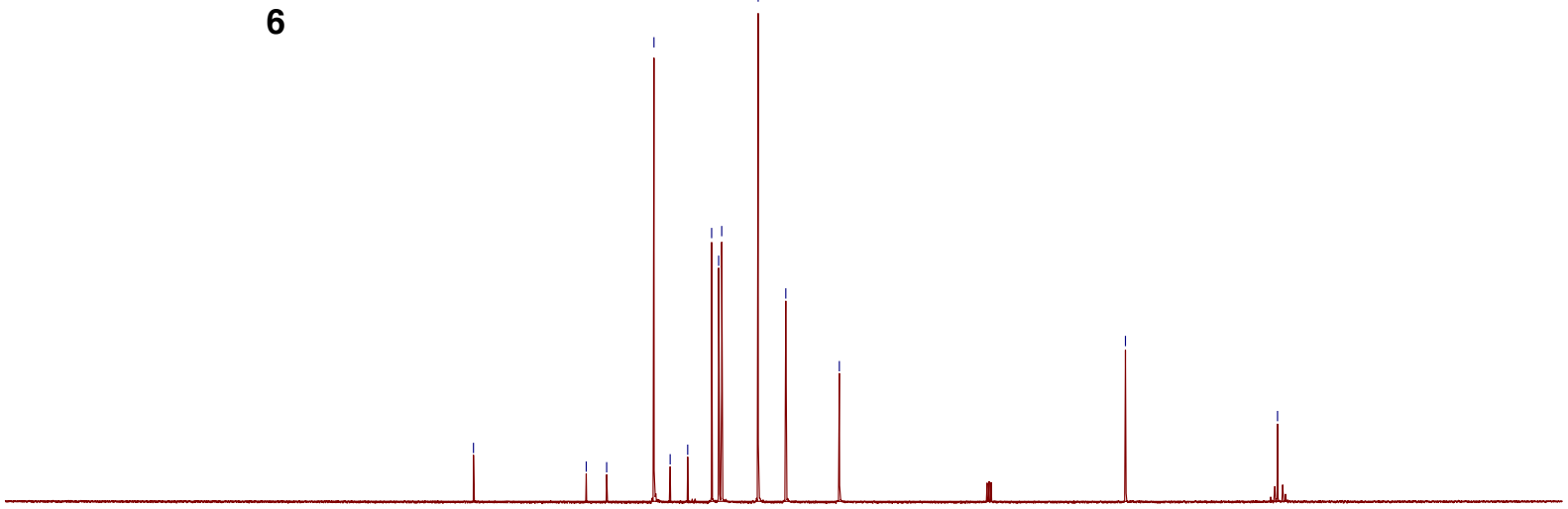

$\begin{array}{lllllllllllllllllllllllllllll}230 & 220 & 210 & 200 & 190 & 180 & 170 & 160 & 150 & 140 & 130 & 120 & 110 & 100 & 90 & 80 & 70 & 60 & 50 & 40 & 30 & 20 & 10 & 0 & -10\end{array}$ 
${ }^{1} \mathrm{H}$ NMR spectrum of 7 in $\mathrm{CDCl}_{3}(400 \mathrm{MHz})$

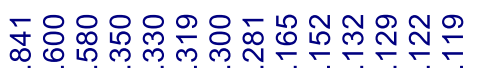

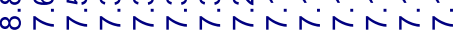

幽

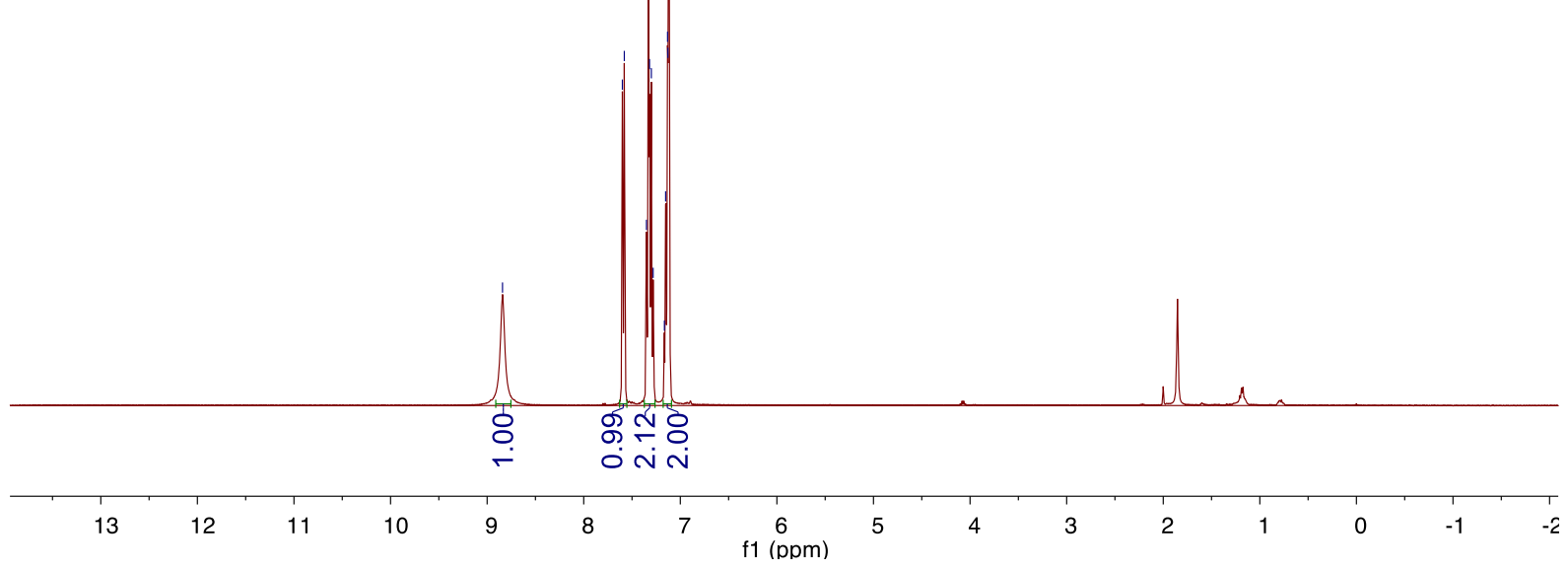

${ }^{13} \mathrm{C}$ NMR spectrum of 7 in $\mathrm{CDCl}_{3}(100 \mathrm{MHz})$

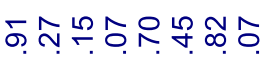

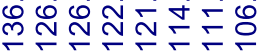

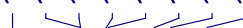<smiles>N#Cc1cc2ccccc2[nH]1</smiles>

$\begin{array}{llllllllllllllllllllllllll}230 & 220 & 210 & 200 & 190 & 180 & 170 & 160 & 150 & 140 & 130 & 120 & 110 & 100 & 90 & 80 & 70 & 60 & 50 & 40 & 30 & 20 & 10 & 0 & -10\end{array}$ 
${ }^{1} \mathrm{H}$ NMR spectrum of 8 in $\mathrm{CDCl}_{3}(400 \mathrm{MHz})$

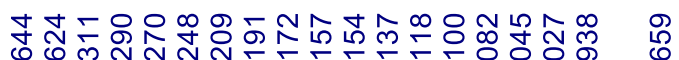

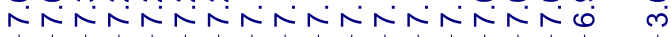

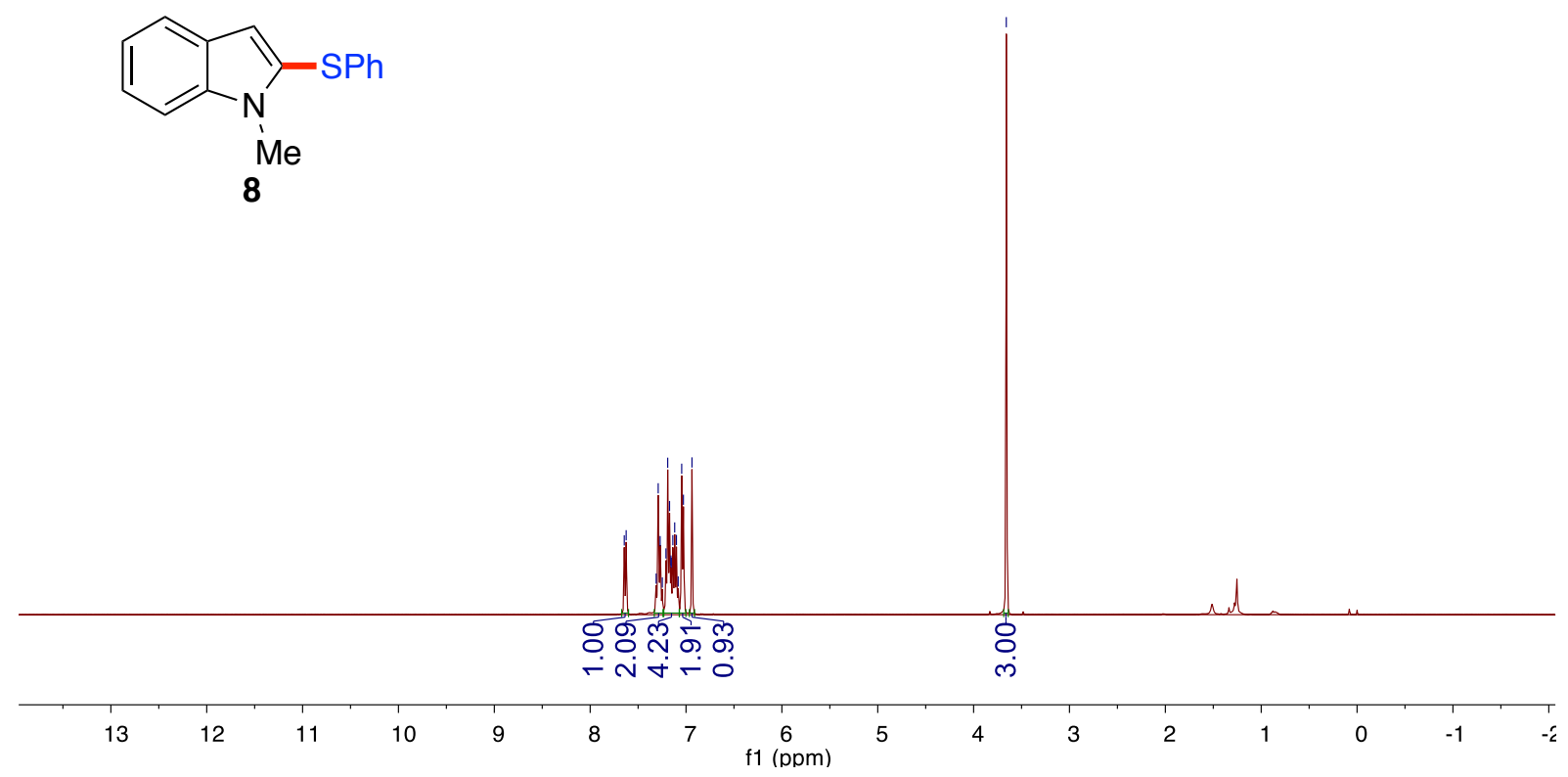

${ }^{13} \mathrm{C}$ NMR spectrum of 8 in $\mathrm{CDCl}_{3}(100 \mathrm{MHz})$

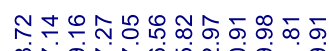

象兹

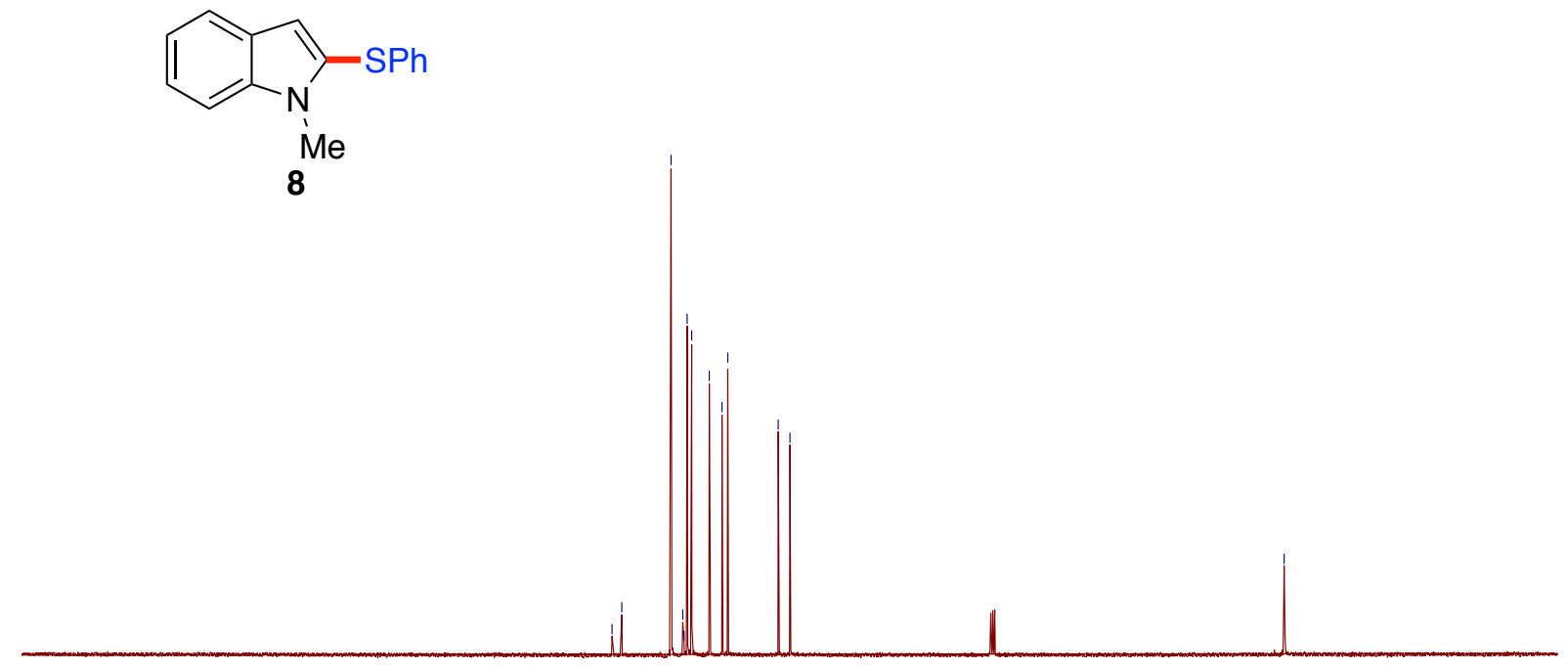

$\begin{array}{llllllllllllllllllllllllll}230 & 220 & 210 & 200 & 190 & 180 & 170 & 160 & 150 & 140 & 130 & 120 & \begin{array}{l}110 \\ 110\end{array} & 100 & 90 & 80 & 70 & 60 & 50 & 40 & 30 & 20 & 10 & 0 & -10\end{array}$ 